\title{
ALMA OBSERVATIONS OF WARM DENSE GAS IN NGC 1614-BREAKING OF THE STAR FORMATION LAW IN THE CENTRAL KILOPARSEC*
}

\author{
C. K. XU ${ }^{1}$, C. CAO $^{1,2,3}$, N. LU ${ }^{1}$, Y. GaO ${ }^{4}$, T. DiaZ-Santos ${ }^{1}$, R. Herrero-Illana ${ }^{5}$, R. Meijerink ${ }^{6}$, G. Privon $^{7}$, Y.-H. ZhaO ${ }^{1,4}$, \\ A. S. Evans ${ }^{7,8}$, S. König ${ }^{9}$, J. M. Mazzarella ${ }^{1}$, S. Aalto ${ }^{10}$, P. Appleton ${ }^{1}$, L. Armus ${ }^{1}$, V. Charmandaris ${ }^{11,12,13}$, J. Chu ${ }^{14}$, \\ S. HaAn $^{15}$, H. InAmi ${ }^{16}$, E. J. Murphy ${ }^{1}$, D. B. SAnders ${ }^{14}$, B. Schulz ${ }^{1}$, And P. van Der WerF ${ }^{6}$ \\ ${ }^{1}$ Infrared Processing and Analysis Center, MS 100-22, California Institute of Technology, Pasadena, CA 91125, USA \\ ${ }^{2}$ School of Space Science and Physics, Shandong University at Weihai, Weihai, Shandong 264209, China \\ ${ }^{3}$ Shandong Provincial Key Laboratory of Optical Astronomy and Solar-Terrestrial Environment, Weihai, Shandong 264209, China \\ ${ }^{4}$ Purple Mountain Observatory, Chinese Academy of Sciences, 2 West Beijing Road, Nanjing 210008, China \\ ${ }_{5}^{5}$ Instituto de Astrofísica de Andalucía - CSIC, Glorieta de la Astronomía, s/n, E-18008 Granada, Spain \\ ${ }^{6}$ Leiden Observatory, Leiden University, P.O. Box 9513, NL-2300 RA Leiden, The Netherlands \\ ${ }^{7}$ Department of Astronomy, University of Virginia, P.O. Box 400325, Charlottesville, VA 22904, USA \\ ${ }^{8}$ National Radio Astronomy Observatory, Charlottesville, VA 22904, USA \\ ${ }^{9}$ Institut de Radioastronomie Millimétrique (IRAM), 300 rue de la Piscine, Domaine Universitaire, F-38406 Saint Martin d'Hères, France \\ ${ }^{10}$ Department of Earth and Space Sciences, Onsala Observatory, Chalmers University of Technology, SE-439 92 Onsala, Sweden \\ ${ }^{11}$ Department of Physics, University of Crete, GR-71003 Heraklion, Greece \\ 12 Institute for Astronomy, Astrophysics, Space Applications \& Remote Sensing, National Observatory of Athens, GR-15236 Penteli, Greece \\ ${ }^{13}$ Chercheur Associé, Observatoire de Paris, F-75014 Paris, France \\ ${ }^{14}$ Institute for Astronomy, University of Hawaii, 2680 Woodlawn Drive, Honolulu, HI 96816, USA \\ ${ }^{15}$ CSIRO Astronomy and Space Science, ATNF, P.O. Box 76, Epping 1710, Australia \\ ${ }^{16}$ National Optical Astronomy Observatory, 950 North Cherry Avenue, Tucson, AZ 85719, USA \\ Received 2014 September 2; accepted 2014 November 4; published 2015 January 12
}

\begin{abstract}
We present ALMA Cycle- 0 observations of the CO (6-5) line emission and of the $435 \mu \mathrm{m}$ dust continuum emission in the central kiloparsec of NGC 1614, a local luminous infrared galaxy at a distance of $67.8 \mathrm{Mpc}$ $\left(1^{\prime \prime}=329 \mathrm{pc}\right)$. The $\mathrm{CO}$ emission is well resolved by the ALMA beam $\left(0^{\prime \prime} 26 \times 0^{\prime} \cdot 20\right)$ into a circumnuclear ring, with an integrated flux of $f_{C O(6-5)}=898( \pm 153) \mathrm{Jy} \mathrm{km} \mathrm{s}^{-1}$, which is $63( \pm 12) \%$ of the total $\mathrm{CO}(6-5)$ flux measured by Herschel. The molecular ring, located between $100 \mathrm{pc}<\mathrm{r}<350 \mathrm{pc}$ from the nucleus, looks clumpy and includes seven unresolved (or marginally resolved) knots with median velocity dispersion of $\sim 40 \mathrm{~km} \mathrm{~s}^{-1}$. These knots are associated with strong star formation regions with $\Sigma_{\mathrm{SFR}} \sim 100 M_{\odot} \mathrm{yr}^{-1} \mathrm{kpc}^{-2}$ and $\Sigma_{\mathrm{Gas}} \sim 10^{4} M_{\odot} \mathrm{pc}^{-2}$. The nondetections of the nucleus in both the $\mathrm{CO}(6-5)$ line emission and the $435 \mu \mathrm{m}$ continuum rule out, with relatively high confidence, a Compton-thick active galactic nucleus in NGC 1614. Comparisons with radio continuum emission show a strong deviation from an expected local correlation between $\Sigma_{\mathrm{Gas}}$ and $\Sigma_{\mathrm{SFR}}$, indicating a breakdown of the Kennicutt-Schmidt law on the linear scale of $\sim 100 \mathrm{pc}$.
\end{abstract}

Key words: galaxies: active - galaxies: evolution - galaxies: general - galaxies: interactions - galaxies: ISM galaxies: nuclei - galaxies: magnetic fields - galaxies: starburst - submillimeter: galaxies

\section{INTRODUCTION}

Luminous infrared galaxies (LIRGs) with $L_{\mathrm{IR}}[8-1000 \mu \mathrm{m}]>$ $10^{11.5} L_{\odot}$, including ultra-luminous infrared galaxies (ULIRGs: $L_{\mathrm{IR}}>10^{12} L_{\odot}$ ), are mostly advanced mergers (Sanders et al. 1988; Sanders \& Mirabel 1996; Scoville et al. 2000; Veilleux et al. 2002). They harbor extreme starbursts (star formation rate (SFR) $\gtrsim 50 \mathrm{M}_{\odot} \mathrm{yr}^{-1}$ ) and sometimes strong active galactic nuclei (AGNs), and are among the most luminous objects in the local universe (Sanders et al. 1988; Genzel et al. 1998; Surace et al. 1998; Veilleux et al. 1999; Scoville et al. 2000; Veilleux et al. 2009). Observations and theoretical simulations have shown that mergers can transform spirals to ellipticals (Toomre 1977; Schweizer 1982; Barnes 1990; Genzel et al. 2001; Veilleux et al. 2002; Dasyra et al. 2006). Gas outflows ubiquitously found in (U)LIRGs (Armus et al. 1990; Heckman et al. 2000; Walter et al. 2002; Rupke et al. 2005; Sakamoto et al. 2009; Fischer et al. 2010; Feruglio et al. 2010; Sturm et al. 2011; Aalto et al. 2012; Veilleux et al. 2013; Cicone et al. 2014) may play an important role in quenching the star formation that

\footnotetext{
* The National Radio Astronomy Observatory is a facility of the National Science Foundation operated under cooperative agreement by Associated Universities, Inc.
}

leads to the formation of red sequence galaxies (Bell et al. 2007; Faber et al. 2007; Hopkins et al. 2008, 2013).

Extensive surveys of $\mathrm{CO}$ rotation lines in low $\mathrm{J}$ transitions such as CO (1-0) at $2.6 \mathrm{~mm}$ and $\mathrm{CO}(2-1)$ at $1.3 \mathrm{~mm}$ have found very large concentrations of molecular gas (up to a few times $10^{10} \mathrm{M}_{\odot}$ ) in the central kiloparsec of (U)LIRGs (Solomon \& Sage 1988; Scoville et al. 1989; Sanders et al. 1991; Solomon et al. 1997; Downes \& Solomon 1998; Bryant \& Scoville 1999; Gao \& Solomon 1999; Evans et al. 2002). This gas, funneled into the nuclear region by the gravitational torque during a merger (Barnes \& Hernquist 1996; Hopkins et al. 2009), provides fuel for the nuclear starburst and/or AGN. However, due to the heavy dust extinction for the UV/optical/near-IR (NIR) observations and the lack of high angular resolution far-IR (FIR)/sub-millimeter (sub-mm)/millimeter ( $\mathrm{mm}$ ) observations, it is still not very clear how the different constituents (i.e., gas, dust, stars, and black holes) in (U)LIRG nuclei interplay with each other. Some studies (Scoville et al. 1997; Downes \& Solomon 1998; Bryant \& Scoville 1999; Gao et al. 2001) suggest that much of the low $\mathrm{J}$ CO luminosities may be due to the emission of diffuse gas not closely related to the active star formation regions. Indeed, single-dish and interferometry $\mathrm{mm}$ and sub-mm observations have found that the intensities 
Table 1

ALMA Observations

\begin{tabular}{|c|c|c|c|c|c|c|c|}
\hline $\begin{array}{l}\text { SB } \\
\text { (1) }\end{array}$ & $\begin{array}{c}\text { Date } \\
\text { (yyyy/mm/dd) } \\
(2)\end{array}$ & Time (UTC) & Config & $N_{\text {ant }}$ & $\begin{array}{r}l_{\max } \\
(\mathrm{m}) \\
(6)\end{array}$ & $\begin{array}{c}t_{\text {int }} \\
\text { (minutes) } \\
(7)\end{array}$ & $\begin{array}{l}T_{\text {sys }} \\
(\mathrm{K}) \\
(8)\end{array}$ \\
\hline X49990a_X505 & $2012 / 08 / 13$ & $11: 31: 46-12: 52: 33$ & $\mathrm{E} \& \mathrm{C}$ & 23 & 402 & 24.7 & 537 \\
\hline X4b58a4_X1ee & $2012 / 08 / 28$ & $08: 58: 50-10: 23: 37$ & $\mathrm{E} \& \mathrm{C}$ & 27 & 402 & 24.7 & 756 \\
\hline
\end{tabular}

Notes. Column 1: schedule-block number; Columns 2 and 3: observation date and time; Column 4: configuration; Column 5: number of antennae; Column 6: maximum baseline length; Column 7: on-target integration time; Column 8: median $T_{\text {sys }}$.

and spatial distributions of star formation in (U)LIRGs correlate significantly stronger with those of higher J CO lines (with upper level $J \geqslant 3$ ), which probe warmer and denser gas than low $J$ lines (Yao et al. 2003; Iono et al. 2004; Wang et al. 2004; Wilson et al. 2008; Iono et al. 2009; Sakamoto et al. 2008; Tsai et al. 2012; Sakamoto et al. 2013; Xu et al. 2014). This is consistent with results of observations of other dense molecular gas indicators such as HCN lines (Solomon et al. 1992; Gao \& Solomon 2004b; Narayanan et al. 2008; Graciá-Carpio et al. 2008; García-Burillo et al. 2012). The multi-J CO observations of Papadopoulos et al. (2012) indicate that for many (U)LIRGs the global CO spectral line energy distribution (SLED) is dominated by a very warm $(T \sim 100 \mathrm{~K})$ and dense $\left(n \geqslant 10^{4} \mathrm{~cm}^{-3}\right)$ gas phase. Lu et al. (2014) found a strong and linear correlation between the mid-J (with upper level J between 5 and 10) luminosity and the $L_{\mathrm{IR}}$ in a Herschel SPIRE Fourier Transform Spectrometer (FTS) survey of a large (U)LIRG sample.

In order to study the warm dense gas in nuclear regions of (U)LIRGs, we observed the CO (6-5) line emission (restframe frequency $=691.473 \mathrm{GHz}$ ) and associated dust continuum emission in two nearby examples, NGC 34 and NGC 1614, using the Band 9 receivers of the Atacama Large Millimeter Array (ALMA; Wootten \& Thompson 2009). Both NGC 34 and NGC 1614 were chosen for these early ALMA observations, among the complete sample of 202 LIRGs of the Great Observatories All-sky LIRG Survey (GOALS; Armus et al. 2009), because of their close proximity $(D<85 \mathrm{Mpc})$ and bright $\mathrm{CO}$ (6-5) line fluxes $\left(f_{\mathrm{CO}(6-5)} \gtrsim 1000 \mathrm{Jy} \mathrm{km} \mathrm{s}^{-1}\right)$ observed in the Herschel SPIRE FTS survey of GOALS galaxies (angular resolution: 30" ; van der Werf et al. 2010; Lu et al. 2014). This enables high signal-to-noise ratio ALMA observations of warm gas structures with linear resolutions of $\lesssim 100 \mathrm{pc}$ for the given angular resolutions of $\sim 0$ ' 25 . Further, both LIRGs have declination angles close to the latitude of the ALMA site, and therefore the Band 9 observations are affected by minimal atmospheric absorption when being carried out near transit.

In this paper, we present ALMA Cycle- 0 observations of the CO (6-5) line emission and the $435 \mu \mathrm{m}$ dust continuum emission in the central kiloparsec of NGC 1614 (also known as Mrk 617 and Arp 186). This LIRG has an infrared luminosity of $L_{\mathrm{IR}}=10^{11.65} L_{\odot}$ (Armus et al. 2009) at a distance of $67.8 \mathrm{Mpc}$ $\left(1^{\prime \prime}=329 \mathrm{pc}\right)$. Most of the current star formation activity is in a circumnuclear starburst ring (Neff et al. 1990; AlonsoHerrero et al. 2001; Díaz-Santos et al. 2008; Olsson et al. 2010), presumably triggered by a minor merger with a mass ratio of ¿ : 1 (Neff et al. 1990; Väisänen et al. 2012). The nucleus itself may harbor a much weaker and older starburst (Alonso-Herrero et al. 2001) and a Compton-thick AGN (Risaliti et al. 2000, but see Olsson et al. 2010; Väisänen et al. 2012). The observations and data reductions are described in Section 2, the results are presented in Section 3, and Section 4 and Section 5 are devoted to a discussion and the summary, respectively. All velocities in this paper are in the radio LSR convention. Throughout this paper, we adopt the $\Lambda$ cosmology with $\Omega_{\mathrm{m}}=0.3$ and $\Omega_{\Lambda}=0.7$, and $H_{0}=70 \mathrm{~km} \mathrm{~s}^{-1} \mathrm{Mpc}^{-1}$.

\section{OBSERVATIONS}

We observed the central region of NGC 1614 in CO (6-5) line emission and $435 \mu \mathrm{m}$ dust continuum emission using the Band 9 receivers of ALMA in the time division mode (TDM; velocity resolution: $6.8 \mathrm{~km} \mathrm{~s}^{-1}$ ). The four basebands (i.e., "Spectral Windows," hereafter SPWs) were centered at the sky frequencies of 680.539, 682.308, 676.826, and $678.764 \mathrm{GHz}$, respectively, each with a bandwidth of $2 \mathrm{GHz}$. Observations were carried out in the extended \& compact (E\&C) configuration using up to 27 antennae (Table 1). The total on-target integration time was 50.4 minutes. During the observations, phase and gain variations were monitored using QSO 0423-013. Observations of the minor planet Ceres were made for the flux calibration. The error in the flux calibration was estimated to be $17 \%$.

The final data reduction was done using CASA 4.1.0. Images were cleaned using Briggs weighting. Both phase and amplitude self-calibrations have been carried out. The primary beam is $\sim 8^{\prime \prime}$. However, emission features larger than $\sim 3^{\prime \prime}$ are poorly sampled because of limited $u v$ coverage for short baselines. Two data sets were generated from the observations. In the first data set, the $\mathrm{CO}(6-5)$ line data cube was generated using data in SPW-0 (sky-freq $=680.539 \pm 1 \mathrm{GHz}$ ), which encompass the $\mathrm{CO}(6-5)$ line emission at the system velocity $\left(4723 \mathrm{~km} \mathrm{~s}^{-1}\right)$ with an effective bandpass of $\sim 800 \mathrm{~km} \mathrm{~s}^{-1}$. The continuum was estimated using data in the other 3 SPWs. In the second data set, the $\mathrm{CO}$ (6-5) line data cube was generated using three SPWs: SPW-0, SPW-1, and SPW-3, respectively. The bandwidth of this CO (6-5) line data cube is $\sim 2400 \mathrm{~km} \mathrm{~s}^{-1}$. For the second data set, the continuum was estimated using data in SPW-2 (skyfreq $=676.826 \pm 1 \mathrm{GHz}$ ). The first data set has better continuum estimation and subtraction, and was therefore used for most of the analysis; the second data set was used to search for evidence of molecular outflows or nuclear $\mathrm{H}^{13} \mathrm{CN}$ (8-7) line emission. The continuum subtraction was carried out using the CASA task UVcontsub. For the first data set, the channel maps of the CO (6-5) line emission have $1-\sigma \mathrm{rms}$ noise of $8.0 \mathrm{mJy}^{-6 a m^{-1}}$ per $6.8 \mathrm{~km} \mathrm{~s}^{-1}$, and for the continuum it is $0.6 \mathrm{mJy}^{\text {beam }}{ }^{-1}$. The CO (6-5) line emission map, integrated over the LSR velocity range between $v=4447.4$ and $4894.5 \mathrm{~km} \mathrm{~s}^{-1}\left(\delta \mathrm{v}=447.1 \mathrm{~km} \mathrm{~s}^{-1}\right)$, has $1 \sigma \mathrm{rms}$ noise of $0.90 \mathrm{Jy}_{\text {beam }}^{-1} \mathrm{~km} \mathrm{~s}^{-1}$. The synthesized beams of these maps are nearly identical, having FWHMs of $0^{\prime \prime} .26 \times 0.20$, corresponding to physical scales of $86 \mathrm{pc} \times 66 \mathrm{pc}$, and a P.A. of $280^{\circ}$. The absolute pointing accuracy of these ALMA observations is on the order of 0.1 . 

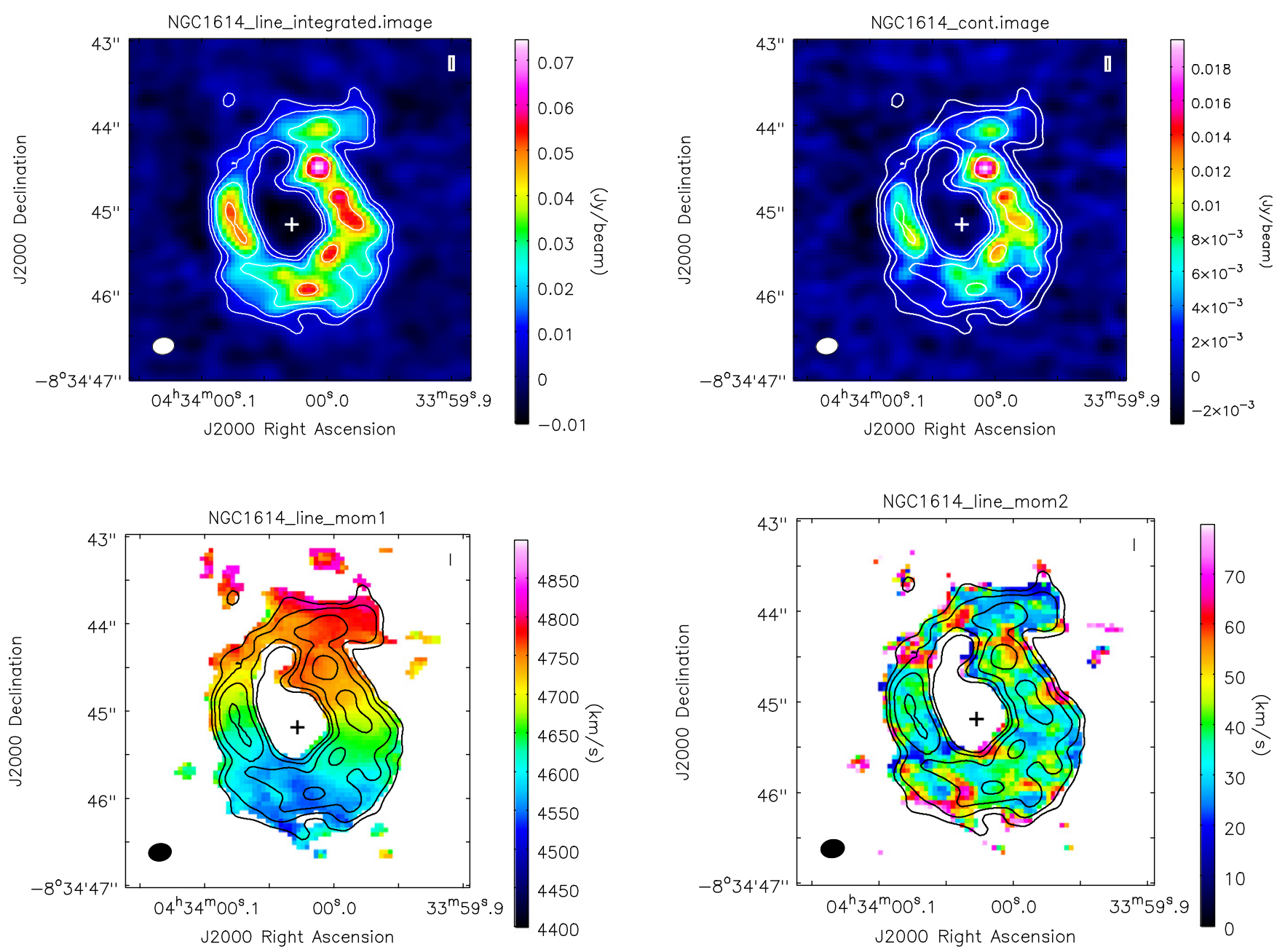

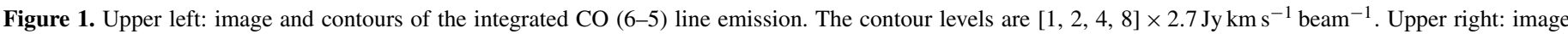

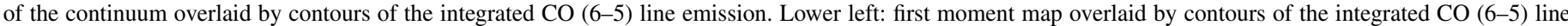

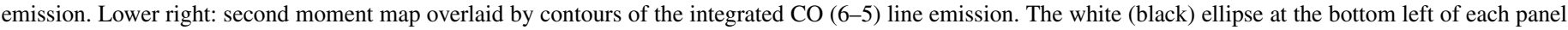

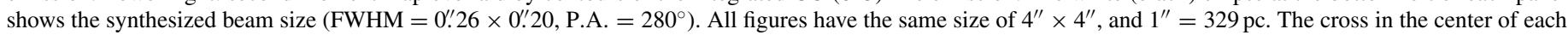
map marks the position of the radio nucleus in the $5 \mathrm{GHz}$ (MERLIN) map (Olsson et al. 2010).

\section{RESULTS}

\subsection{The CO (6-5) Line Emission}

In Figure 1, we present images of the integrated $\mathrm{CO}(6-5)$ line emission, the continuum at $435 \mu \mathrm{m}$, the first moment map, and the second moment map. All images are overlaid by the same contours of the integrated $\mathrm{CO}(6-5)$ line emission at levels of $[1,2,4,8] \times 2.7 \mathrm{Jy} \mathrm{beam}^{-1} \mathrm{~km} \mathrm{~s}^{-1}$. The line and the continuum emissions correlate closely with each other, both showing a ring configuration without a detectable nucleus. The ring has a diameter of $\sim 2^{\prime \prime}(\sim 650 \mathrm{pc})$. In both the line and the continuum maps, the ring looks clumpy and can be decomposed into several knots. The first moment map shows a clear velocity gradient along the south-north direction, consistent with an inclined rotating ring. According to Olsson et al. (2010), the inner disk of NGC 1614 has an inclination angle of $51^{\circ}$. In the second moment map, the velocity dispersion in most regions in the ring is rather constant at the level of $\delta \mathrm{v} \sim 40 \mathrm{~km} \mathrm{~s}^{-1}$, though in some inter-knot regions it can be as low as $\delta \mathrm{v} \sim 20 \mathrm{~km} \mathrm{~s}^{-1}$. From the first moment map, the velocity gradient due to the rotation can be estimated to be $d V / d r \sim 0.3 \mathrm{~km} \mathrm{~s}^{-1} \mathrm{pc}^{-1}$, corresponding to a line widening of $\sim 20 \mathrm{~km} \mathrm{~s}^{-1}$ within individual beams (linear size: $\sim 80 \mathrm{pc}$ ). This is consistent with the lowest velocity dispersion seen in the second moment map.

The channel maps $\left(\delta \mathrm{v}=20.4 \mathrm{~km} \mathrm{~s}^{-1}\right)$ are shown in Figure 2, overlaid on the image of the integrated line emission. They provide more details about the rotating ring. First of all, given the relatively narrow local velocity dispersions (see the second moment map in Figure 1), the channel maps dissect the ring spatially. It appears that the spatial width of ring segments in individual channel maps are generally broader than that in the integrated emission map. This is because, by co-adding all channel maps, the integrated emission map is affected more severely by the (negative) sidelobes of different segments of the ring. This is a significant effect because some ring segments are separated by $\sim 3^{\prime \prime}$, the angular scale limit of our interferometer observations. Indeed the total flux of the $\mathrm{CO}(6-5)$ line emission, $f_{\mathrm{CO}(6-5)}=898( \pm 153) \mathrm{Jy} \mathrm{km} \mathrm{s}^{-1}$ (the error being dominated by the calibration uncertainty) that is derived from the sum of the aperture photometry of individual channels (centered on the emission features for each given channel), is $31 \%$ higher than that measured on the integrated $\mathrm{CO}(6-5)$ line emission map. Comparison with the Herschel measurement of the integrated 


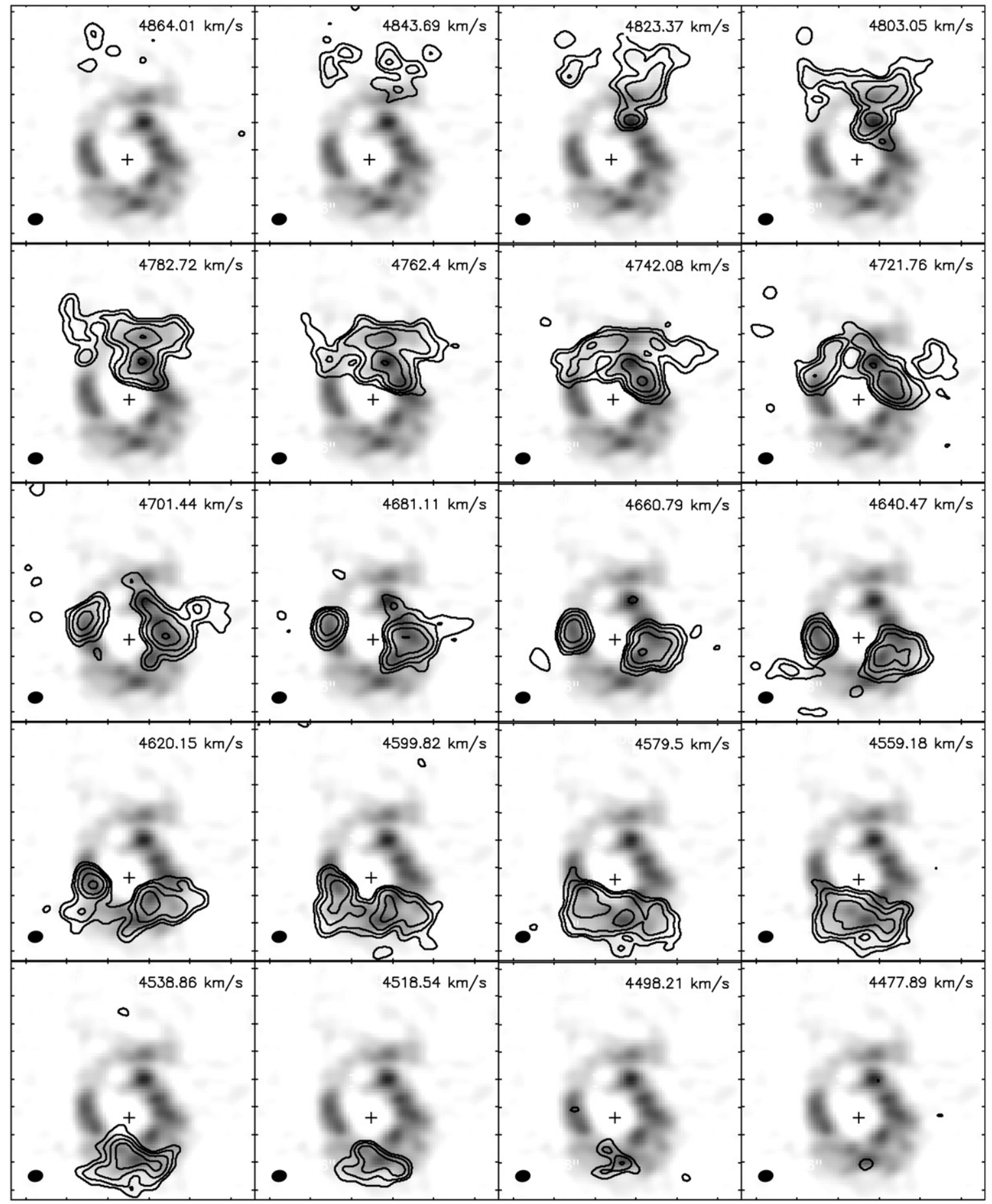

Figure 2. $\mathrm{CO}(6-5)$ line emission contours of the channel maps (the velocity channel width $=20.4 \mathrm{~km} \mathrm{~s}^{-1}$ ), and overlaid on the integrated emission map. The contour levels are $21 \mathrm{mJy}$ beam $^{-1} \times[1,2,4,8,16]$. All maps have the same size of $4^{\prime \prime} \times 4^{\prime \prime}$. In each panel, the central velocity of the channel is given. (The system velocity of NGC 1614 is $4723 \mathrm{~km} \mathrm{~s}^{-1}$.)

CO (6-5) line emission of NGC $1614\left(1423 \pm 126 \mathrm{Jy} \mathrm{km} \mathrm{s}^{-1}\right.$ within a beam of $\sim 30^{\prime \prime}$; van der Werf et al. 2010; Lu et al. 2014) yields an interferometer-to-single-dish flux ratio of $0.63 \pm 0.12$. This suggests that most warm dense gas in NGC 1614 is concentrated in the circumnuclear ring.

Figure 3 shows plots of the velocity distributions of the central region (radius $=1^{\prime \prime} .5 \simeq 500 \mathrm{pc}$ ) and of the peak position (R.A. $=04^{\mathrm{h}} 34^{\mathrm{m}} 00^{\mathrm{s}} .006$, decl. $=-08^{\circ} 34^{\prime} 44^{\prime \prime} .47$ ) of the integrated $\mathrm{CO}(6-5)$ line emission map. In order to reduce the noise, we used relatively broad bins of $\delta \mathrm{v}=34 \mathrm{~km} \mathrm{~s}^{-1}$.
The velocity distribution has a FWHM of $272 \mathrm{~km} \mathrm{~s}^{-1}$. Its shape is rather irregular and spiky, reflecting the clumpiness of the rotating ring and the narrow velocity dispersions of individual clumps (Figure 1). No evidence for any outflow/inflow of $|\delta \mathrm{v}|<1200 \mathrm{~km} \mathrm{~s}^{-1}$, nor any detection of the $\mathrm{H}^{13} \mathrm{CN}(8-7)$ line (rest-frame frequency $=690.552 \mathrm{GHz}$ ), can be found in the spectrum. In the velocity distribution of the peak position, which is in the northwestern quadrant of the ring (Figure 1), we also found no evidence of outflow/inflow or of the $\mathrm{H}^{13} \mathrm{CN}(8-7)$ line emission. 


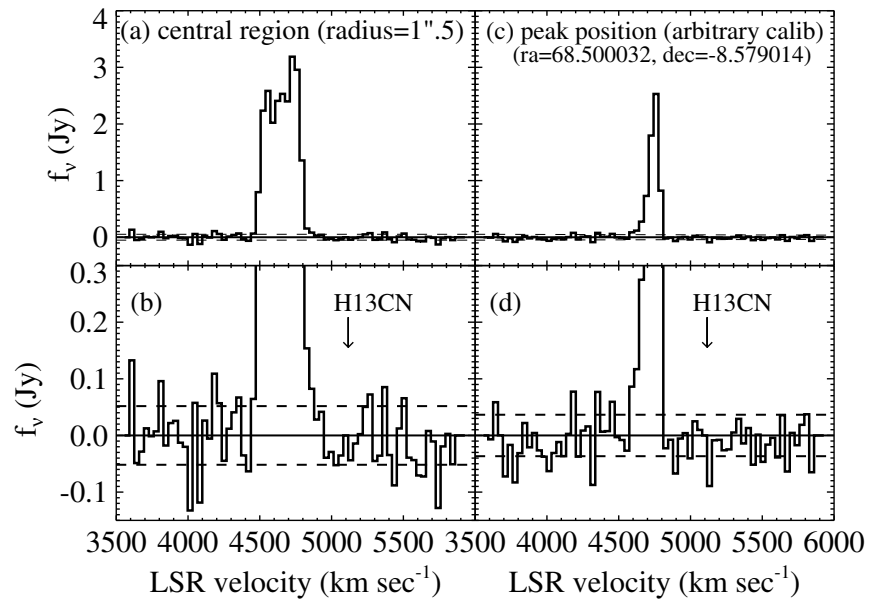

Figure 3. Panel (a): spectrum of the CO (6-5) line emission in the velocity domain, measured in the channel maps with an aperture of radius $=1$ '.5. The dashed lines mark the $1 \sigma$ noise boundaries. Panel (b): zoom-in of the bottom part of panel (a). Again the dashed lines mark the $1 \sigma$ noise boundaries. The arrow marks the expected location of the $\mathrm{H}^{13} \mathrm{CN}(8-7)$ line at the systemic velocity of $v=4723 \mathrm{~km} \mathrm{~s}^{-1}$. Panel (c): spectrum at the peak position (R.A. $=04: 34: 00.006$, decl. $=-08: 34: 44.47)$ of the integrated CO (6-5) line emission map. In order to show it more clearly, the flux is scaled up arbitrarily. Panel (d): zoom-in of the bottom part of panel (c).

\subsection{The $435 \mu \mathrm{m}$ Continuum Emission}

The flux density of the $435 \mu \mathrm{m}$ continuum measured by ALMA is $f_{435 \mu \mathrm{m}}=269 \pm 46 \mathrm{mJy}$. The continuum correlates spatially with the $\mathrm{CO}(6-5)$ emission in the central kiloparsec of NGC 1614 (Figure 1). This suggests that dust heating and gas heating in the warm dense gas cores are strongly coupled, a conclusion also reached by Lu et al. (2014) in a Herschel FTS study of the CO SLED of LIRGs. NGC 1614 was observed by Herschel-SPIRE (Griffin et al. 2010) both in the photometry mode (J. Chu et al., in preparation) and in the FTS mode (van der Werf et al. 2010; Lu et al. 2014), with beams of $\sim 30^{\prime \prime}$. Because the error of the continuum measured in the FTS mode is large $(\sim 1 \mathrm{Jy})$, we estimated the total flux of the $435 \mu \mathrm{m}$ continuum of NGC 1614 using SPIRE photometer fluxes $f_{350 \mu \mathrm{m} \text {,SPIRE }}=1916 \pm 134 \mathrm{mJy}$ and $f_{500 \mu \mathrm{m} \text {,SPIRE }}=487 \pm 34 \mathrm{mJy}$. Assuming a power-law spectrum for the dust continuum (i.e., $\log \mathrm{f}_{v}$ depending on $\log v$ linearly), we carried out linear interpolation in the logarithmic domain of the flux and of the frequency between 350 and $500 \mu \mathrm{m}$, and found $f_{435 \mu \mathrm{m}, \text { SPIRE }}=831 \pm 58 \mathrm{mJy}$. The ratio between $f_{435 \mu \mathrm{m}}$ and $f_{435 \mu \mathrm{m}, \text { SPIRE }}$ then yields an interferometer-to-single-dish flux ratio of $0.32 \pm 0.06$. This is a factor of $\sim 2$ lower than the interferometer-to-single-dish flux ratio of the line emission, indicating that the distribution of dust is substantially more extended than that of the warm dense gas.

The total dust mass in NGC 1614 can be estimated using the mid- and far-IR fluxes in the Spitzer/MIPS $24 \mu \mathrm{m}$ band and in the Herschel 70, 100, 160, 250, 350, and $500 \mu \mathrm{m}$ bands. The Herschel data are taken from J. Chu et al. (in preparation). A least-squares fit to the IR SED by a twograybody model, with the emissivity spectral index $\beta=2$ for both components, yields a total dust mass of $M_{\text {dust,total }}=$ $10^{7.60 \pm 0.07} M_{\odot}$ with a cold dust temperature of $T_{C}=35 \pm 2 \mathrm{~K}$ (Figure 4). Fits by two-graybody models with $\beta$ as a free parameter or by the model of Draine \& Li (2007) yield very similar results. If dust in the central region has the same $T_{C}$, then $M_{\text {dust,cent }}=f_{435 \mu \mathrm{m}, \mathrm{ALMA}} / f_{435 \mu \mathrm{m}, \mathrm{wSPIRE}} \times M_{\text {dust,total }}$. Taking

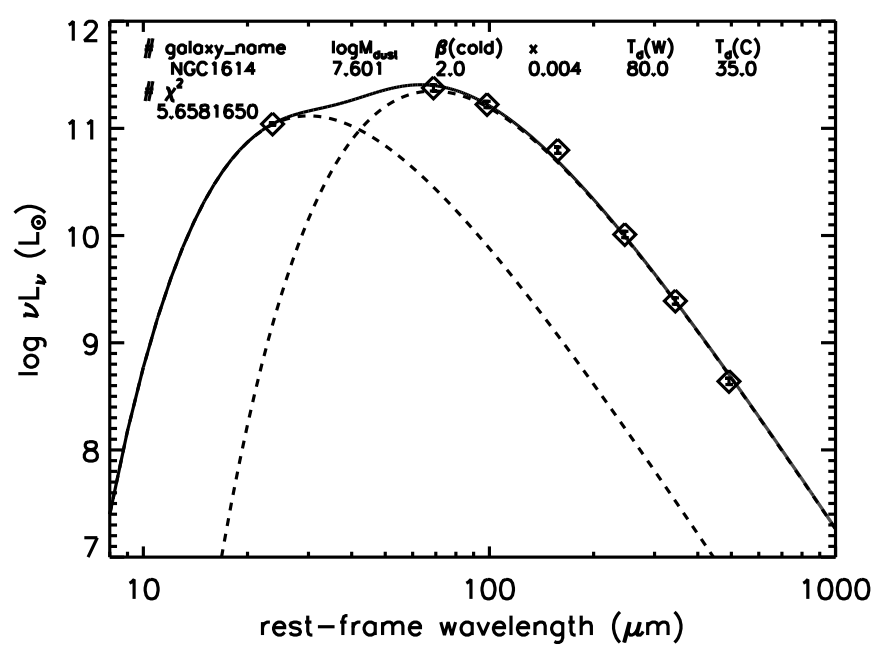

Figure 4. SED fitting of the dust emission in NGC 1614 by a two-graybody model.

into account the uncertainties due to the assumption on the cold dust temperature $(\sim 50 \%)$, the dust mass in the central region observed by ALMA is $M_{\text {dust,cent }}=10^{7.11 \pm 0.20} M_{\odot}$. NGC 1614 has a metallicity of $12+\log (\mathrm{O} / \mathrm{H})=8.65 \pm 0.10$ (Armus et al. 1989; Vacca \& Conti 1992; Engelbracht et al. 2008; Modjaz et al. 2011). According to Rémy-Ruyer et al. (2014), for galaxies with $12+\log (\mathrm{O} / \mathrm{H})>8.5$, the gas-to-dust ratio is 100 with a $1-\sigma$ uncertainty of $\sim 0.2$ dex. Therefore, assuming $M_{\text {gas }} / M_{\text {dust }}=10^{2.0 \pm 0.2}$, the gas mass in the central region of NGC 1614 is $M_{\text {gas,cent }}=10^{9.11 \pm 0.30} M_{\odot}$. This is consistent with the molecular gas mass (which should dominate the total gas mass) found in the same region $\left(M_{\text {gas,cent }}=10^{9.30} M_{\odot}\right.$, with a conversion factor of $X_{\mathrm{CO}}=3 \times 10^{20} \mathrm{~cm}^{-2}\left(\mathrm{~K} \mathrm{~km} \mathrm{~s}^{-1}\right)^{-1}$; König et al. 2013). It should be pointed out that both the ALMA continuum observations and the SMA observations of CO (2-1) by König et al. (2013) detected mostly dust and gas emission in dense gas structures and missed significantly the diffuse emission; therefore, the dust and gas mass derived from these observations are lower limits.

There has been a debate on whether there is an AGN in NGC 1614. Risaliti et al. (2000) argued, based on the detection of a hard X-ray source and its spectrum, that in the center of NGC 1614 there is a hidden AGN obscured by Comptonthick gas $\left(N_{H}>1.5 \times 10^{24} \mathrm{~cm}^{-2}\right.$; Comastri 2004). However, such high column density gas in the nucleus, which will not be affected by the missing flux issue, is not detected in either the $\mathrm{CO}$ (6-5) map or the dust continuum map. Using the scaling factor between the gas mass and the continuum flux derived above, the non-detection of the continuum in the nucleus $\left(\sigma=0.6 \mathrm{mJy}\right.$ beam $\left.^{-1}\right)$ sets a $3 \sigma$ upper limit for the gas surface density of $N_{H}=10^{23.1 \pm 0.3} \mathrm{~cm}^{-2}$. Since an AGN cannot hide itself in the emission of dust that absorbs its UV/optical/NIR radiation, our results can rule out this possibility with relatively high confidence. Indeed a Compton-thick torus with a radius of $r=20$ pc (García-Burillo et al. 2014), which fills $23 \%$ of the ALMA beam, should be detectable in the continuum with a signal-to-noise ratio of $s / \sigma \geqslant 7$. The $s / \sigma$ could be even higher since the $T_{d}$ in a torus is likely much warmer than the assumed dust temperature of $T_{d}=35 \mathrm{~K}$. The high-resolution MIR $L$-band observations of Väisänen et al. (2012) also argue against a Compton-thick AGN in NGC 1614. As pointed out by Olsson et al. (2010) and Herrero-Illana et al. (2014), the X-ray source 

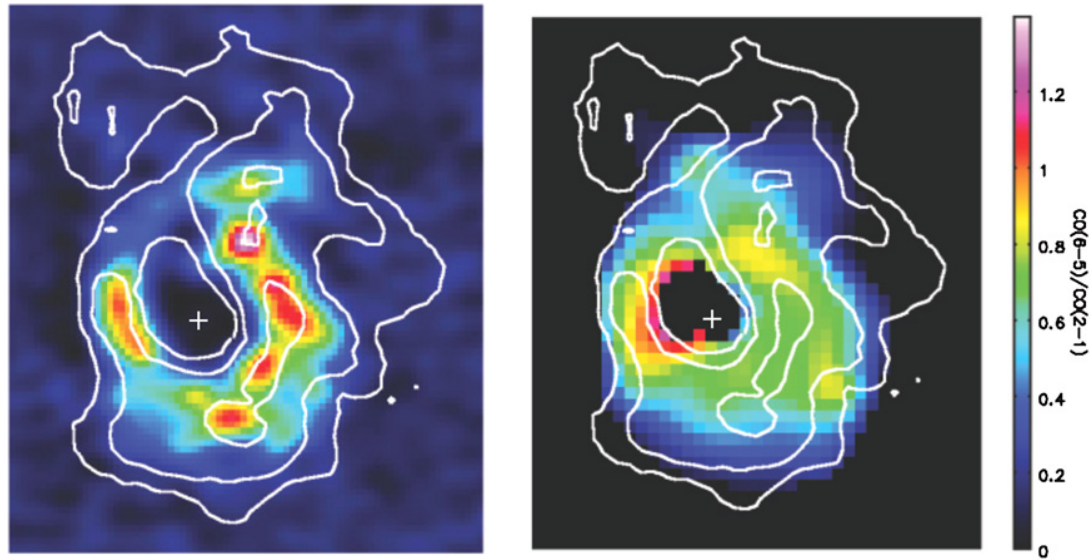

Figure 5. Left: comparison between integrated CO (6-5) line emission map (resolution: $0^{\prime \prime} 26 \times 0$ 0!20) and integrated CO (2-1) line emission contours (resolution: 0'.50 × 0.'44; König et al. 2013). Right: contours of integrated CO (2-1) line emission overlaid on image of the ratio between integrated CO (6-5) line emission and integrated CO (2-1) line emission, with both being smoothed to a common beam (convolution of two original beams). Signals in the both maps are in the same units of $\mathrm{K} \mathrm{km} \mathrm{s}^{-1}$

detected by Risaliti et al. (2000) could be explained by low-mass $\mathrm{X}$-ray binaries.

\section{DISCUSSION}

\subsection{Comparison with Previous CO Observations}

There is rich literature on molecular line observations in the sub-mm and mm bands for NGC 1614 (Young et al. 1986; Solomon \& Sage 1988; Scoville et al. 1989; Sanders et al. 1991; Casoli et al. 1991; Gao \& Solomon 2004a; Albrecht et al. 2007; Wilson et al. 2008; Olsson et al. 2010; Costagliola et al. 2011; König et al. 2013; Imanishi \& Nakanishi 2013). The single dish CO (1-0) observations of Sanders et al. (1991) found a total molecular gas mass of $10^{10.12} M_{\odot}$, assuming a standard conversion factor of $X_{\mathrm{CO}}=3 \times 10^{20} \mathrm{~cm}^{-2}\left(\mathrm{~K} \mathrm{~km} \mathrm{~s}^{-1}\right)^{-1}$ and distance of $D=67.8 \mathrm{Mpc}$. This is consistent with the result of Casoli et al. (1991), but significantly larger than those obtained in earlier and less sensitive observations (Young et al. 1986; Solomon \& Sage 1988). The OVRO observations of Scoville et al. (1989), with a beam of $4^{\prime \prime} \times 6^{\prime \prime}$, allocated $30 \%$ of the total $\mathrm{CO}(1-0)$ emission within a nuclear region of radius $=1 \mathrm{kpc}$. The more recent and higher-resolution $(2$ ". $75 \times 2$ ". 40$)$ observations of Olsson et al. (2010) resolved the central CO (1-0) line emission into an arc-like feature $\sim 3 \mathrm{kpc}$ in length and $\sim 1.3 \mathrm{kpc}$ in width, but did not resolve the ring. The SMA map of CO $(3-2)$ (beam $=2$ ". $6 \times 2$ ". 1 ; Wilson et al. 2008) and the ALMA maps of $\mathrm{HCN} / \mathrm{HCO}^{+} / \mathrm{HNC}(4-3)\left(\right.$ beam $=1^{\prime \prime} .5 \times 1$ 1". 3 ; Imanishi $\&$ Nakanishi 2013) also did not resolve the ring.

Before our ALMA observations, the best angular resolution for any CO rotation lines was obtained by König et al. (2013) in their SMA observations of the CO (2-1) line, with a beam of $0.50 \times 0$.'44. In Figure 5, we compare their CO (2-1) map with our CO (6-5) map. In the ring the two maps have good correspondence, though the $\mathrm{CO}(6-5)$ emission looks clumpier, most likely due to the better angular resolution. König et al. (2013) noticed a strong asymmetry in the CO (2-1) distribution between the eastern and western sides of the ring, and interpret it as a consequence of the feeding of the ring by the dust lane on the northwest of the ring. In the CO (6-5) map, we still see this asymmetry albeit being less prominent than in CO (2-1). When smoothed to a common beam, the ratio between the two emissions is rather constant in most regions of the ring, with a median brightness temperature ratio of 0.72 (Figure 5). The east quadrant of the ring has the highest brightness temperature ratio $(\sim 1)$. This could be due, at least partially, to a slight mismatch between the two maps, given the steep gradient in both maps in this region. On the other hand, this seems to be consistent with the stronger east-west asymmetry seen in the CO (2-1) map than in the CO (6-5) map. König et al. (2013) argued that the reason of the asymmetry could be the feeding of the ring by a dust lane on the northwest of the ring. In this scenario, the east quadrant has higher $\mathrm{CO}(6-5) / \mathrm{CO}(2-1)$ ratio than the west quadrant because it has less diffuse gas (freshly fed by the dust lane) compared to the west quadrant. The nucleus is not detected in either map. The CO (6-5) 3- $\sigma$ upper limit of $742 M_{\odot} \mathrm{pc}^{-2}$ $\left(N_{H}=10^{22.86} \mathrm{~cm}^{-2}\right)$ for the surface density of the warm dense gas, derived by assuming the same relation between $\Sigma_{\mathrm{Gas}}$ and $\mathrm{CO}$ (6-5) surface brightness in the ring region (Equation (3)), is consistent with the upper limit set by the $435 \mu \mathrm{m}$ continuum. If the conversion factor advocated by Downes \& Solomon (1998) for (U)LIRGs is used, which is a factor of $\sim 6$ lower than the standard value adopted in Equation (3), the result is a significantly lower value for the upper limit of $\Sigma_{\text {Gas }}$ in the nucleus. In the region north of the ring, where significant $\mathrm{CO}$ (2-1) emission is found, little CO (6-5) emission is detected and the brightness temperature ratio is $<0.1$.

\subsection{Relation between Warm Dense Gas and Star Formation}

Most star formation in NGC 1614 is occurring in the circumnuclear starburst ring. Soifer et al. (2001) found that $72 \%$ of the $12 \mu \mathrm{m}$ flux of NGC 1614 measured by IRAS is contained within the $2^{\prime \prime}$ beam of their Keck observations. The comparison between the high-resolution $8.4 \mathrm{GHz}$ map of the central kiloparsec region (Herrero-Illana et al. 2014) and that of a lower-resolution map at the same frequency by Schmitt et al. (2006) indicates that $\sim 67 \%$ of the total SFR is contributed by the starburst ring and the nucleus. The star formation in the ring has been studied by Alonso-Herrero et al. (2001) using a Hubble Space Telescope (HST) Pa $\alpha$ map, Díaz-Santos et al. (2008) using a Gemini $8 \mu \mathrm{m}$ map, Väisänen et al. (2012) using a $3.3 \mu \mathrm{m}$ polycyclic aromatic hydrocarbon (PAH) map obtained with UIST (an imager-spectrometer for integral field spectroscopy) at UKIRT, Olsson et al. (2010) and Herrero-Illana et al. (2014) using VLA and Merlin radio continuum maps. While the NIR and MIR observations may still be affected by the dust obscuration associated with the dense gas (Imanishi \& Nakanishi 2013), the radio continuum is an SFR indicator insensitive to dust obscuration. 

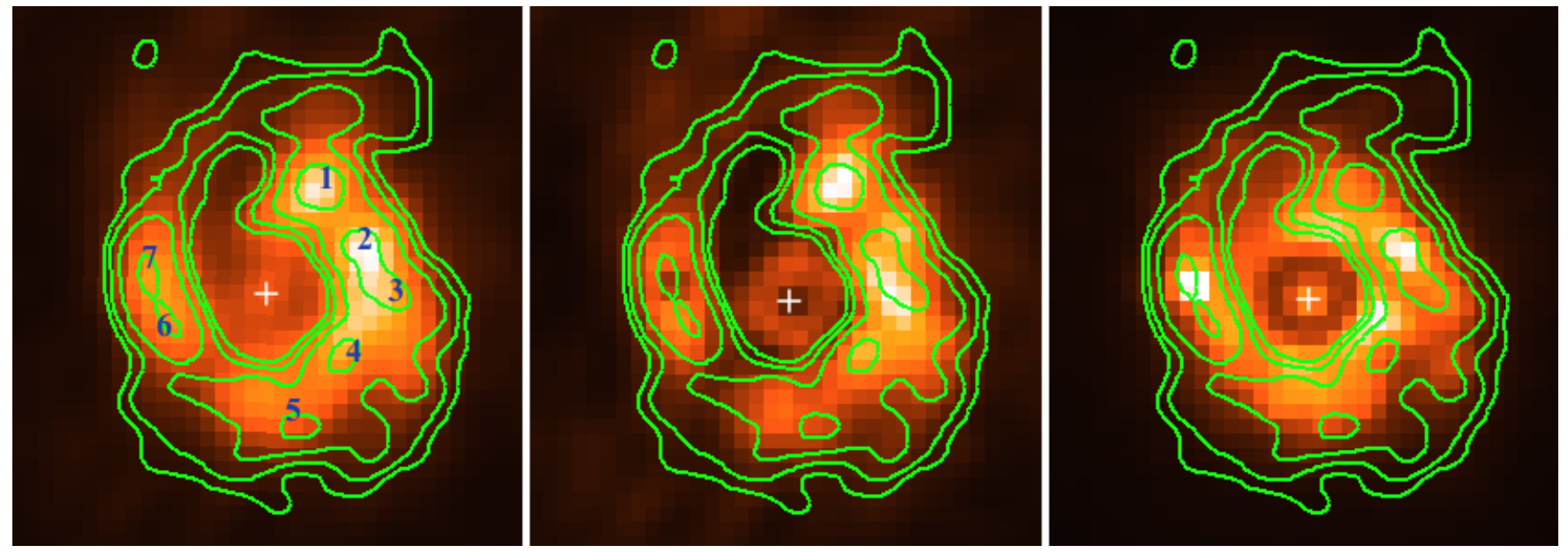

Figure 6. Comparison between contours of the integrated $\mathrm{CO}$ (6-5) line emission and images of the total $8.4 \mathrm{GHz}$ radio continuum (left), the nonthermal radio component (middle), and the thermal radio component (right). Positions of $\mathrm{CO}(6-5)$ knots listed in Table 2 are marked by corresponding numbers in the left panel.

In the left panel of Figure 6, we compare the CO (6-5) contours with the radio continuum at $8.4 \mathrm{GHz}$ (beam = $0 ! 41 \times 0$ '.26, Herrero-Illana et al. 2014). The CO (6-5) knots in the ring (Table 2) are marked in the image. While there is a radio nucleus, the $\mathrm{CO}$ map has a hole at the ring's center. Herrero-Illana et al. (2014) argued that the radio nucleus is not an AGN, which is consistent with our conclusion that there is no (Compton-thick) AGN in NGC 1614 (Section 3.2). In the other two panels of Figure 6, the CO (6-5) emission is compared to the thermal and nonthermal radio emission components, respectively. Following Herrero-Illana et al. (2014), the thermal radio emission is estimated using a high-resolution, extinctioncorrected Pa- $\alpha$ map obtained from a set of HST NIR narrowand broadband images. The method involves the comparison of a Pa- $\alpha$ equivalent width map as well as an NIR color (F160W/F222M) image to stellar population synthesis models (Starburst99; Leitherer et al. 1999) to derive a spatially resolved dust obscuration map with which to correct the original $\mathrm{Pa}-\alpha$ image (see also Díaz-Santos et al. 2008). The details of this procedure can be found in the Appendix. As a final step, the nonthermal component is derived by subtracting from the total radio emission the thermal component. The thermal fraction of the radio continuum at $8.4 \mathrm{GHz}$ is found to be $51 \%$ in the ring region $(100<\mathrm{r}<350 \mathrm{pc})$.

In Figure 7, the radial profiles of the emissions are compared. The peak of the radial distribution of the thermal radio is shifted (by $\sim 70 \mathrm{pc}$ ) toward the smaller radius compared to that of the $\mathrm{CO}(6-5)$ radial distribution. The peak of the distribution of the total radio emission also has a small offset compared to that of the $\mathrm{CO}(6-5)$, while the radial profile of nonthermal radio emission is similar to that of the $\mathrm{CO}(6-5)$ in the ring region. The radio profile of CO (2-1) (König et al. 2013) is also shown in the same plot. If we compare the $\mathrm{CO}(2-1)$ profile with the profile of $\mathrm{CO}(6-5)$, which has been smoothed to the same resolution of $\mathrm{CO}(2-1)$, we see that the former is significantly more extended than the latter. Beyond $r \sim 400 \mathrm{pc}$, the cold and diffuse molecular gas probed by CO (2-1) emission is devoid of any significant star formation (as revealed by the profiles of the radio emissions).

In Figure 8, we plot the SFR surface density $\left(\Sigma_{\mathrm{SFR}}\right)$ versus the gas surface density $\left(\Sigma_{\text {Gas }}\right.$; i.e., the Kennicutt-Schmidt law) for the nuclear starburst and individual cells (3 pixels, pixel $=00^{\prime}(089)$ in the ring, using the thermal and nonthermal

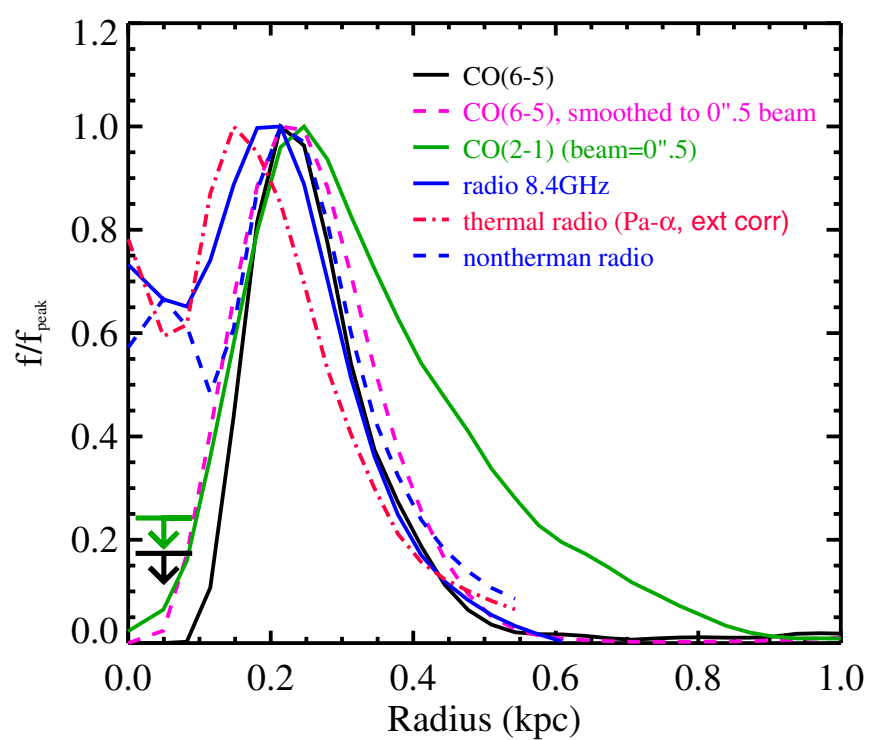

Figure 7. Comparison between normalized radial profiles of the $\mathrm{CO}(6-5)$, total radio continuum at $8.4 \mathrm{GHz}$, nonthermal radio component, thermal radio component, and $\mathrm{CO}(2-1)$. The arrows at $r=0.1 \mathrm{kpc}$ show the $3 \sigma$ upper limits of $\mathrm{CO}(6-5)$ and $\mathrm{CO}(2-1)$ in the central hole.

maps to derive $\Sigma_{\mathrm{SFR}}$ and the CO (6-5) map (smoothed and regridded to match the radio maps) to obtain $\Sigma_{\text {Gas. }}$. The SFR can be estimated from the nonthermal and thermal radio luminosities using two formulae given in Murphy et al. (2012), respectively:

$$
\left(\frac{\mathrm{SFR}_{v}^{\mathrm{nth}}}{M_{\odot} \mathrm{yr}^{-1}}\right)=6.64 \times 10^{-29}\left(\frac{v}{\mathrm{GHz}}\right)^{\alpha^{\mathrm{nth}}}\left(\frac{\mathrm{L}_{v}^{\mathrm{nth}}}{\operatorname{erg~s}^{-1} \mathrm{~Hz}^{-1}}\right) .
$$

and

$$
\begin{aligned}
\left(\frac{\mathrm{SFR}_{v}^{\text {th }}}{M_{\odot} \mathrm{yr}^{-1}}\right)= & 4.6 \times 10^{-28}\left(\frac{\mathrm{T}_{\mathrm{e}}}{10^{4} \mathrm{~K}}\right)^{-0.45} \\
& \times\left(\frac{v}{\mathrm{GHz}}\right)^{0.1}\left(\frac{\mathrm{L}_{v}^{\text {th }}}{\mathrm{erg} \mathrm{s}^{-1} \mathrm{~Hz}^{-1}}\right),
\end{aligned}
$$

where $T_{e}=10^{4} \mathrm{~K}, v=8.4 \mathrm{GHz}$, and $\alpha^{\text {nth }}=1.2$ (HerreroIllana et al. 2014). For individual cells in the ring, we also 
Table 2

CO (6-5) Knots in Circumnuclear Starburst Ring

\begin{tabular}{|c|c|c|c|c|c|c|c|}
\hline (1) & (2) & (3) & (4) & (5) & (6) & (7) & (8) \\
\hline ID & $\begin{array}{c}\text { R.A. } \\
\text { (J2000) }\end{array}$ & $\begin{array}{c}\text { Decl. } \\
(\mathrm{J} 2000)\end{array}$ & $\begin{array}{c}S_{\mathrm{CO}(6-5)} \\
\left(\mathrm{Jy} \mathrm{km} \mathrm{s}^{-1} \text { beam }^{-1}\right)\end{array}$ & $\begin{array}{c}S_{435 \mu \mathrm{m}} \\
\left(\mathrm{mJy} \mathrm{beam}^{-1}\right)\end{array}$ & $\begin{array}{c}\log \left(\Sigma_{\mathrm{Gas}}\right) \\
\left(M_{\odot} \mathrm{pc}^{-2}\right)\end{array}$ & $\begin{array}{c}\log \left(\Sigma_{\mathrm{SFR}}\right) \\
\left(M_{\odot} \mathrm{yr}^{-1} \mathrm{kpc}^{-2}\right)\end{array}$ & Notes \\
\hline 1 & 04:34:00.006 & $-08: 34: 44.49$ & $34.0 \pm 6.1$ & $19.6 \pm 3.5$ & 3.94 & 2.49 & 4 \\
\hline 2 & 04:33:59.991 & $-08: 34: 44.86$ & $27.2 \pm 4.9$ & $13.8 \pm 2.5$ & 3.87 & 2.40 & 6 \\
\hline 3 & $04: 33: 59.981$ & $-08: 34: 45.10$ & $25.5 \pm 4.6$ & $12.1 \pm 2.2$ & 3.91 & 2.33 & 6 \\
\hline 4 & 04:33:59.998 & $-08: 34: 45.52$ & $25.5 \pm 4.6$ & $11.7 \pm 2.1$ & 3.88 & 2.41 & 7 \\
\hline 5 & 04:34:00.015 & $-08: 34: 45.95$ & $25.7 \pm 4.6$ & $7.7 \pm 1.4$ & 3.87 & 2.14 & 8 \\
\hline 6 & 04:34:00.069 & $-08: 34: 45.29$ & $22.8 \pm 4.1$ & $10.3 \pm 1.9$ & 3.81 & 2.36 & 10 \\
\hline 7 & 04:34:00.077 & $-08: 34: 45.04$ & $23.6 \pm 4.2$ & $8.2 \pm 1.5$ & 3.84 & 2.00 & 10 \\
\hline
\end{tabular}

Notes. Column 4: CO (6-5) peak flux; Column 5: continuum peak flux; Column 6: peak molecular gas surface density, after smoothed to the beam of 8.4 GHz observations $(0$. '41 × 0.'26); Column 7: peak SFR density, derived using flux of the nonthermal radio at 8.4 GHz; Column 8: corresponding GMA in König et al. (2013).

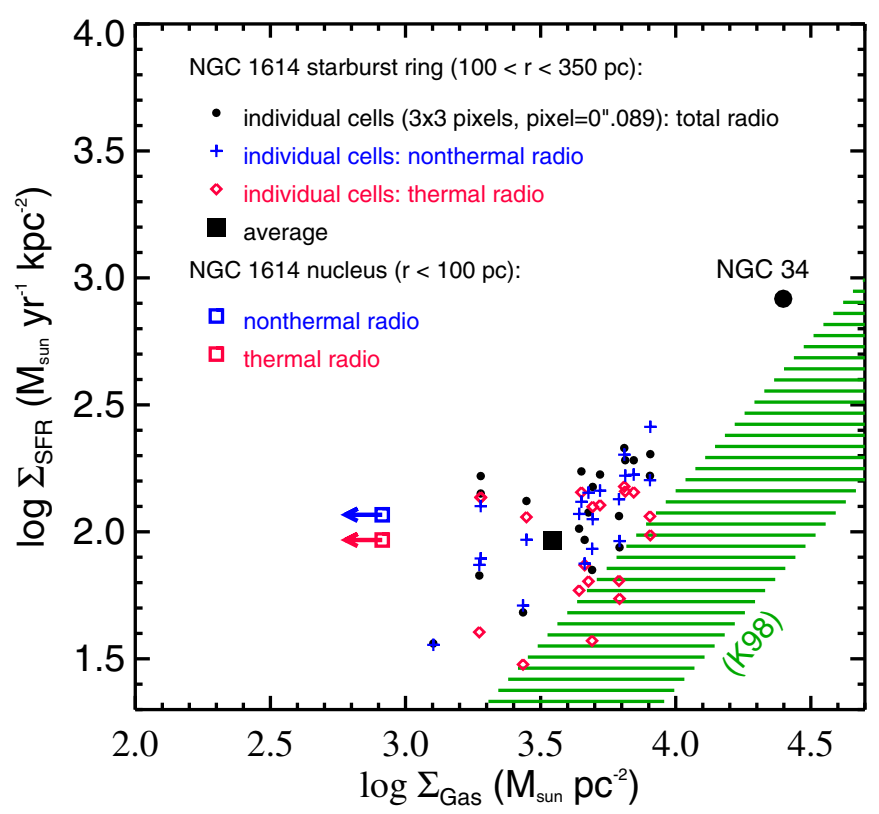

Figure 8. Plot of $\log \Sigma_{\mathrm{SFR}}$ vs. $\log \Sigma_{\mathrm{Gas}}$. For individual cells in the NGC 1614 ring, $\log \Sigma_{\text {SFR,th }}$ (red diamonds) and $\log \Sigma_{\text {SFR,nth }}$ (blue crosses), and $\log \Sigma_{\text {SFR,total }}$ (black dots) are estimated using the thermal, nonthermal, and total radio maps, respectively; and $\log \Sigma_{\mathrm{Gas}}$ is estimated from the $\mathrm{CO}(6-5)$ map that is smoothed and regridded to match the radio maps. For the NGC 1614 nucleus (open squares), the $3 \sigma$ upper limit for $\Sigma_{\mathrm{Gas}}$ was derived using the $\mathrm{CO}$ (6-5) map assuming the same relation for the ring region (Equation (3)). The average for the NGC 1614 ring (black solid square): data taken from Table 3. Nuclear starburst in NGC 34 (black solid circle): data taken from Table 3. The shaded area (in green color) represents the data for local starbursts in the sample of Kennicutt (1998).

plotted in Figure 8 the $\Sigma_{\mathrm{SFR}}$ versus $\Sigma_{\mathrm{Gas}}$ relation with the $\Sigma_{\mathrm{SFR}}$ estimated from the total radio emission, assuming a constant nonthermal fraction $\left(\mathrm{f}_{\mathrm{nth}}=0.5\right)$ and the SFR versus $L^{\text {nth }}$ relation in Equation (1).

The gas surface density was estimated using the CO (6-5) surface brightness as follows. According to König et al. (2013), the total $\mathrm{H}_{2}$ mass in the ring is $M_{\mathrm{H}_{2}}=10^{8.97} M_{\odot}$ (for $D=67.8 \mathrm{Mpc}$ ), estimated using the CO (1-0) map of Olsson et al. (2010) and assuming a conversion factor of $3 \times 10^{20} \mathrm{~cm}^{-2}\left(\mathrm{~K} \mathrm{~km} \mathrm{~s}^{-1}\right)^{-1}$. Dividing this by the integrated $\mathrm{CO}(2-1)$ flux of the ring, $S_{\mathrm{CO}(2-1)}=65.4 \pm 6.9 \mathrm{Jy} \mathrm{km} \mathrm{s}^{-1}$ (König et al. 2013) and assuming a brightness temperature ratio of 0.72 between $\mathrm{CO}(6-5)$ and $\mathrm{CO}$ (2-1) (Figure 5), we have

$$
\left(\frac{\Sigma_{\mathrm{Gas}}}{M_{\odot} \mathrm{pc}^{-2}}\right)=20.3 \times\left(\frac{f_{\mathrm{CO}(6-5)}}{{\mathrm{Jy} \operatorname{arcsec}^{-2} \mathrm{~km} \mathrm{~s}^{-1}}}\right) .
$$

In the ring region, only cells that are detected in both radio and $\mathrm{CO}(6-5)$ maps above a 3- $\sigma$ threshold are plotted (therefore the random errors are $<0.12$ dex for these data points). In the nuclear region $(r<100 \mathrm{pc})$, the $3 \sigma$ upper limit for $\Sigma_{\text {Gas }}$ was derived using the $\mathrm{CO}$ (6-5) map assuming the same relation for the ring region (Equation (3)). For individual cells in the ring, the $\Sigma_{\mathrm{SFR}}$ versus $\Sigma_{\mathrm{Gas}}$ relation is systematically above that for local starbursts (Kennicutt 1998), indicating a higher star formation efficiency (SFE). This is because, by relating the SFR to the warm dense gas probed by the highresolution ALMA observations of CO (6-5), much of the cold diffuse gas probed by low J CO (more extended than the warm dense gas) is excluded from the $\Sigma_{\text {Gas }}$ in our results. It is worth noting that we used a standard $\mathrm{CO}$ conversion factor $\left(X_{\mathrm{CO}}=3 \times 10^{20} \mathrm{~cm}^{-2}\left(\mathrm{~K} \mathrm{~km} \mathrm{~s}^{-1}\right)^{-1}\right)$ for NGC 1614 data. In the literature, arguments for high SFE in (U)LIRGs are very often based on results obtained using a $\mathrm{CO}$ conversion factor $\sim 5$ times lower than the standard value (e.g., Daddi et al. 2010; Genzel et al. 2010).

It appears that on the linear scale of $100 \mathrm{pc}$, the tight correlation previously found between $\Sigma_{\mathrm{SFR}}$ versus $\Sigma_{\mathrm{Gas}}$ (Kennicutt 1998; Genzel et al. 2010; Leroy et al. 2013; Yao et al. 2003; Iono et al. 2004; Wilson et al. 2008) breaks down in the central kiloparsec of NGC 1614. In particular, the non-detections of the nucleus in both $\mathrm{CO}(6-5)$ and $\mathrm{CO}(2-1)$ maps set a lower-limit of the $\Sigma_{\mathrm{SFR}}$-to- $\Sigma_{\mathrm{Gas}}$ ratio about an order of magnitude above the nominal value, corresponding to a very short gas exhaustion timescale of $M_{\text {gas }} / \mathrm{SFR}<10 \mathrm{Myr}$. The low extinction (Alonso-Herrero et al. 2001; Kotilainen et al. 2001; Díaz-Santos et al. 2008) and low PAH emission (Väisänen et al. 2012) also indicate an ISM depression in the nuclear region. The star formation timescale associated with the thermal radio is $\sim 10$ Myr and that with the nonthermal radio is $\sim 100 \mathrm{Myr}$. Alonso-Herrero et al. (2001) argued that, based on detections of deep CO stellar absorption, NGC 1614 harbors a nuclear starburst older than $10 \mathrm{Myr}$, which could have blown away the ambient ISM (Väisänen et al. 2012). If the timescale for the feedback effects, including both the gas consumption by star formation and mass loss by superwinds, is significantly shorter than 10 Myr (the dynamic timescale of the nuclear region is only $\tau \sim 1 \mathrm{Myr}$ ), then the deviation of the nucleus from the $\Sigma_{\mathrm{SFR}}$ versus $\Sigma_{\mathrm{Gas}}$ relation may indeed be due to the feedback of 
the old nuclear starburst. This is consistent with the results of García-Burillo et al. (2012) who found that NGC 1614 has the highest value of the SFE (estimated from the FIR/HCN ratio) among a sample of normal star-forming galaxies and mergers, and argued that this could be due to the exhaustion of the dense molecular gas by starburst activity.

In the starburst ring, the correlation between $\Sigma_{\mathrm{SFR} \text {,th }}$ and $\Sigma_{\mathrm{Gas}}$ is rather weak (Spearman's rank correlation coefficient $\rho=0.37$ with the significance of its deviation from zero $p=0.20$ ). Also, the correlation between $\Sigma_{\mathrm{SFR} \text {,total }}$ (estimated using total radio emission) and $\Sigma_{\text {Gas }}$ has a large scatter, and is only marginally significant $(\rho=0.64$ and $p=0.0023)$. This is consistent with the systematic offset between the radial profiles of the total radio and $\mathrm{CO}(6-5)$ in Figure 7 . While the weak correlation between $\Sigma_{\text {SFR,th }}$ and $\Sigma_{\text {Gas }}$ could be mainly due to the uncertainties associated with the obscuration correction of the $\mathrm{Pa} \alpha$ (the thermal radio is estimated using the obscuration-corrected $\mathrm{Pa} \alpha$ ), this cannot explain the lack of correlation between $\Sigma_{\mathrm{SFR} \text {,total }}$ and $\Sigma_{\text {Gas }}$. We have already seen a breakdown of the $\Sigma_{\text {SFR }}-$ to- $-\Sigma_{\text {Gas }}$ correlation in the nucleus, and interpreted it as a consequence of starburst feedback. The same scenario can be applied to the individual cells in the ring. Given the high resolutions of the radio and $\mathrm{CO}(6-5)$ maps, these cells correspond to star formation regions of linear scales of $\sim 100 \mathrm{pc}$. On such fine scales, the $\Sigma_{\mathrm{SFR}}-$ to $-\Sigma_{\mathrm{Gas}}$ relation could be sensitive to the local star-formation history. Indeed, Alonso-Herrero et al. (2001) suggested that in NGC 1614 the starburst propagates like a "wild fire" from the nucleus outward. Väisänen et al. (2012) proposed that even the ring is stratified in terms of the star formation age. In a CO (1-0) survey of M33, Onodera et al. (2010) found a breakdown of the Kennicutt-Schmidt law on the linear scale of $\sim 80 \mathrm{pc}$, and attributed it to the various evolutionary stages of giant molecular clouds (GMCs) and to the drift of young clusters from their parent GMCs. These interpretations are applicable to our results, although our ALMA observations probe an even tighter correlation between $\mathrm{CO}(6-5)$ and SFR in a LIRG associated with a starburst merger.

A stronger correlation is found between $\Sigma_{\mathrm{SFR}, \text { nth }}$ and $\Sigma_{\mathrm{Gas}}$ in the starburst ring $\left(\rho=0.81\right.$ and $\left.p=1.6 \times 10^{-5}\right)$. This is puzzling because, given the longer star formation timescale associated with the nonthermal radio, this relation should be more sensitive to the star formation history than the $\Sigma_{\mathrm{SFR}, \text { th }}$ and $\Sigma_{\text {Gas }}$ relation. It is likely that the $\Sigma_{\mathrm{SFR}, \text { nth }}$ and $\Sigma_{\mathrm{Gas}}$ correlation is driven by other factors than the Kennicutt-Schmidt law. One possibility is that it is due to the correlation between the magnetic field strength and the gas density (Fiebig \& Guesten 1989; Helou \& Bicay 1993; Niklas \& Beck 1997). Observationally, this correlation extends from the smallest (Fiebig \& Guesten 1989) to the largest cosmic scales (Vallée 1990, 1995), and has the form of $B \propto n^{k}$ for $n>10^{2} \mathrm{~cm}^{-3}$, where $\mathrm{B}$ is the magnetic field strength, $\mathrm{n}$ the gas density, and $k=0.5 \pm 0.1$ (Fiebig \& Guesten 1989). Since the emissivity of the nonthermal (synchrotron) radiation is proportional to $\mathrm{B}^{2}$, the $B$-versus- $n$ correlation leads naturally to a localized ( $\sim$ linear) correlation between nonthermal surface brightness and gas surface density. Another possibility is that the nonthermal radio and the $\mathrm{CO}(6-5)$ correlate with each other because they are both powered by cosmic rays (CRs). Indeed, CSO observations of ${ }^{12} \mathrm{CO}(6-5)$ and ${ }^{13} \mathrm{CO}(6-5)$ by Hailey-Dunsheath et al. (2008) of the nuclear starburst in the central 180 pc of NGC 253 suggested that warm molecular gas is most likely to be heated by an elevated density of CRs or by turbulence. In order to test whether CRs dominate the heating of warm molecular gas in NGC 1614, we carried out model-fitting using theoretical models (Meijerink \& Spaans 2005; Kazandjian et al. 2012, 2014) of the cosmic ray dominated regions (CDRs) and photon dominated regions (PDRs) to fit the emission lines of ${ }^{12} \mathrm{CO},{ }^{13} \mathrm{CO}, \mathrm{HCN}, \mathrm{HNC}$, and $\mathrm{HCO}^{+}$. These are mostly single dish data for the entire system of NGC 1614 (Sanders et al. 1991; Albrecht et al. 2007; Costagliola et al. 2011; N. Lu et al. 2014, in preparation), plus some high-resolution interferometry data for the central region taken from the literature (Wilson et al. 2008; Olsson et al. 2010; König et al. 2013; Imanishi \& Nakanishi 2013) and from this work. The results show that PDR models with strong mechanical heating (by turbulence) provide the best fit while CDR models fit the data rather poorly. Details of these results will be presented elsewhere (R. Meijerink et al., in preparation). This is consistent with Rosenberg et al. (2014a) who modeled the Herschel observations of ${ }^{12} \mathrm{CO}$ up to upper $J=13$ and ${ }^{13} \mathrm{CO}$ up to upper $J=6$, together with data of other sub-mm lines taken from the literature, of NGC 253. They found that mechanical heating by turbulence is necessary to reproduce the observed molecular emission and CR heating is a negligible heating source. Rosenberg et al. (2014b) reached a similar conclusion for Arp 299A, a nuclear starburst in Arp 299 (a merger-induced LIRG). In principle, the turbulence can be related to the CRs through shocks generated by supernova remnants (SNRs) that can both power the turbulence (Draine 1980) and accelerate CRs (Drury et al. 1994). However, given the very different mechanisms for energizing low-velocity turbulence and for CR acceleration by SNR shocks, it is unlikely that this can explain the localized correlation between $\Sigma_{\mathrm{SFR}, \mathrm{nth}}$ and $\Sigma_{\mathrm{Gas}}$ in the starburst ring down to the linear scale of $100 \mathrm{pc}$.

\subsection{NGC 1614 and NGC 34: A Tale of Two LIRGs}

In this section, we compare NGC 1614 with NGC 34, another local LIRG observed by our team using ALMA band-9 receivers (Xu et al. 2014). Both galaxies are late-stage mergers (Neff et al. 1990; Schweizer \& Seitzer 2007). As shown in Table 3, they have similar absolute $K$-band magnitude $M_{K}$ (indicating similar stellar mass), similar total gas mass as obtained by $\mathrm{H}$ i and $\mathrm{CO}$ observations, and similar total SFR as derived from the IR+UV luminosities (U et al. 2012). On the other hand, as revealed by the ALMA observations and high angular resolution observations in other bands, the two galaxies are very different in the central kiloparsec.

First of all, our ALMA data ruled out a Compton-thick AGN in NGC 1614. By comparison, there is a weak AGN in NGC 34 according to the X-ray data (Brightman \& Nandra 2011; Esquej et al. 2012), and the ALMA results (Xu et al. 2014) are consistent with the AGN being Compton thick. Nevertheless, for both galaxies, the central kiloparsec is dominated by starburst activity and AGN contributions to both dust and gas heatings are insignificant ( $\mathrm{Xu}$ et al. 2014; Stierwalt et al. 2013). In NGC 34, the starburst is concentrated in a compact nuclear disk of $r \sim 100 \mathrm{pc}$, with very high $\Sigma_{\mathrm{SFR} \text {,th }}$ and $\Sigma_{\mathrm{Gas}}$. In NGC 1614, a starburst ring between $r_{\text {in }}=100 \mathrm{pc}$ and $r_{\text {out }}=350 \mathrm{pc}$ dominates the central region, with moderate mean $\Sigma_{\mathrm{SFR} \text {,th }}$ and mean $\Sigma_{\mathrm{Gas}}$ compared to other local starbursts (Figure 8). It is worth pointing out that different $\mathrm{CO}$ conversion factors have been adopted for the two cases: for the nuclear starburst in NGC 34, the ALMA observations showed that the molecular gas is concentrated in a well organized disk controlled mostly by the gravity of stars $(\mathrm{Xu}$ et al. 2014). Therefore, we choose to use the conversion factor for (U)LIRGs: $X_{\mathrm{CO}}=0.5 \times 10^{20} \mathrm{~cm}^{-2}\left(\mathrm{~K} \mathrm{~km} \mathrm{~s}^{-1}\right)^{-1}$ (Scoville et al. 1997; Downes \& Solomon 1998). On the other hand, 
Table 3

Comparison between NGC 1614 and NGC 34

\begin{tabular}{|c|c|c|}
\hline & NGC 1614 & NGC 34 \\
\hline$\overline{\text { R.A. }(\text { J2000) }})^{a}$ & $04^{\mathrm{h}} 34^{\mathrm{m}} 00^{\mathrm{s}} .03$ & $00^{\mathrm{h}} 11^{\mathrm{m}} 06^{\mathrm{s}} .54$ \\
\hline Decl. $(\mathrm{J} 2000)^{\mathrm{a}}$ & $-08^{\circ} 34^{\prime} 45^{\prime \prime} .1$ & $-12^{\circ} 06^{\prime} 27^{\prime \prime} .5$ \\
\hline Distance (Mpc) & 67.8 & 84.1 \\
\hline$L_{\mathrm{IR}}\left(L_{\odot}\right)^{\mathrm{b}}$ & $10^{11.65}$ & $10^{11.49}$ \\
\hline$M_{K}(\mathrm{mag})^{\mathrm{c}}$ & -24.59 & -24.46 \\
\hline$M_{\mathrm{HI}}\left(M_{\odot}\right)^{\mathrm{d}}$ & $10^{9.45}$ & $10^{9.72}$ \\
\hline $\operatorname{SFR}_{\text {tot }}\left(M_{\odot} \mathrm{yr}^{-1}\right)^{\mathrm{e}}$ & 51.3 & 34.7 \\
\hline$M_{\mathrm{H}_{2}, \text { tot }}\left(M_{\odot}\right)^{\mathrm{f}}$ & $10^{10.12}$ & $10^{10.15}$ \\
\hline$M_{\text {dust,tot }}\left(M_{\odot}\right)^{\mathrm{g}}$ & $10^{7.60}$ & $10^{7.48}$ \\
\hline $\mathrm{AGN}$ & No & Yes \\
\hline Merger mass ratio & $4: 1-5: 1$ & $3: 2-3: 1$ \\
\hline$S_{8.4 \mathrm{GHz}, \mathrm{tot}}(\mathrm{mJy})^{\mathrm{h}}$ & 41.1 & \\
\hline$S_{\mathrm{CO}(6-5), \text { tot }}\left(\mathrm{Jy} \mathrm{km} \mathrm{s}^{-1}\right)^{\mathrm{i}}$ & $1423 \pm 126$ & $937 \pm 63$ \\
\hline$S_{435 \mu \mathrm{m}, \text { tot }}(\mathrm{mJy})^{\mathrm{j}}$ & $831 \pm 58$ & $517 \pm 36$ \\
\hline \multicolumn{3}{|l|}{ Central Starburst } \\
\hline Morphology & Circumnuclear ring & Nuclear disk \\
\hline Radius (pc) & $r_{\text {in }}=100, r_{\text {out }}=350$ & 100 \\
\hline$S_{8.4 \mathrm{GHz}, \text { cent }}(\mathrm{mJy})^{\mathrm{k}}$ & 26.5 & 15.2 \\
\hline $\operatorname{SFR}_{\text {cent }}\left(\mathrm{M}_{\odot} \mathrm{yr}^{-1}\right)^{1}$ & 32.8 & 26.0 \\
\hline$\Sigma_{\mathrm{SFR}}\left(M_{\odot} \mathrm{yr}^{-1} \mathrm{kpc}^{-2}\right)^{\mathrm{m}}$ & 92.8 & 827.6 \\
\hline$M_{\mathrm{H}_{2}, \text { cent }}\left(M_{\odot}\right)^{\mathrm{n}}$ & $10^{8.97}$ & $10^{8.76}$ \\
\hline$\Sigma_{\mathrm{Gas}}\left(M_{\odot} \mathrm{pc}^{-2}\right)^{\mathrm{o}}$ & $10^{3.54}$ & $10^{4.40}$ \\
\hline$S_{\mathrm{CO}(6-5), \text { cent }}\left(\mathrm{Jy} \mathrm{km} \mathrm{s}^{-1}\right)^{\mathrm{p}}$ & $898 \pm 153$ & $1004 \pm 151$ \\
\hline$S_{435 \mu \mathrm{m}, \mathrm{cent}}(\mathrm{mJy})^{\mathrm{q}}$ & $269 \pm 46$ & $275 \pm 41$ \\
\hline$M_{\text {dust,cent }}\left(M_{\odot}\right)^{\mathrm{r}}$ & $10^{7.11}$ & $10^{6.97}$ \\
\hline
\end{tabular}

Notes.

a Coordinates of the nucleus in the $8.4 \mathrm{GHz}$ radio continuum.

b IR luminosity between 8-1000 $\mu \mathrm{m}$ (Armus et al. 2009).

c Absolute $K$-band magnitude (Rothberg \& Joseph 2004).

$\mathrm{d}$ Total mass of neutral atomic hydrogen gas, taken from compilation by Kandalyan (2003).

e Total star formation rate (U et al. 2012).

$\mathrm{f}$ Total mass of molecular hydrogen gas (assuming $X_{\mathrm{CO}}=3 \times$ $\left.10^{20} \mathrm{~cm}^{-2}\left(\mathrm{~K} \mathrm{~km} \mathrm{~s}^{-1}\right)^{-1}\right)$; NGC 1614: Sanders et al. (1991); NGC 34: Kruegel et al. (1990).

g Total dust mass; NGC 1614: this work; NGC 34: Esquej et al. (2012).

h Total flux of the $8.4 \mathrm{GHz}$ radio continuum (Schmitt et al. 2006).

i Total flux of the CO (6-5) emission (N. Lu et al. 2014, in preparation).

$\mathrm{j}$ Total flux of the $435 \mu \mathrm{m}$ continuum emission; NGC 1614: this work; NGC 34: $\mathrm{Xu}$ et al. (2014).

k Flux of the $8.4 \mathrm{GHz}$ radio continuum in the central region; NGC 1614: Herrero-Illana et al. (2014); NGC 34: Condon et al. (1991).

1 The SFR of central starburst: $\mathrm{SFR}_{\text {cent }}=\mathrm{SFR}_{\text {tot }} \times \mathrm{f}_{\text {cent }}$, where $f_{\text {cent }}=$ $S_{8.4 \mathrm{GHz}, \text { cent }} / S_{8.4 \mathrm{GHz}, \text { tot }}=0.64$ for NGC 1614 , and $f_{\text {cent }}=0.75$ for NGC 34 .

${ }^{m}$ Mean SFR column density of the central starburst.

${ }^{\mathrm{n}}$ Mass of molecular hydrogen gas in the central region; NGC $1614\left(X_{\mathrm{CO}}=\right.$ $\left.3 \times 10^{20} \mathrm{~cm}^{-2}\left(\mathrm{~K} \mathrm{~km} \mathrm{~s}^{-1}\right)^{-1}\right)$ : König et al. (2013); NGC $34\left(X_{\mathrm{CO}}=0.5 \times\right.$ $\left.10^{20} \mathrm{~cm}^{-2}\left(\mathrm{~K} \mathrm{~km} \mathrm{~s}^{-1}\right)^{-1}\right)$ : Fernandez et al. (2014).

${ }^{\circ}$ Mean gas column density of the central starburst $\left(M_{\mathrm{Gas}}=1.36 \times M_{\mathrm{H}_{2}}\right)$.

p Flux of the $\mathrm{CO}(6-5)$ emission in the central starburst region; NGC 1614: this work; NGC 34: Xu et al. (2014).

q Flux of the $435 \mu \mathrm{m}$ continuum emission in the central starburst region; NGC 1614: this work; NGC 34: Xu et al. (2014).

${ }^{\mathrm{r}}$ Dust mass in the central starburst region; NGC 1614: this work; NGC 34: Xu et al. (2014).

for the starburst ring in NGC 1614, the ALMA observations presented here and the SMA observations of König et al. (2013) reveal that much of the $\mathrm{CO}$ emission is clumped in individual knots associated with giant molecular associations (GMAs), which might be self-gravitating. In this case, a standard Galactic $\mathrm{CO}$ conversion factor is more appropriate (Papadopoulos et al. 2012): $X_{\mathrm{CO}}=3.0 \times 10^{20} \mathrm{~cm}^{-2}\left(\mathrm{~K} \mathrm{~km} \mathrm{~s}^{-1}\right)^{-1}$. Nevertheless,

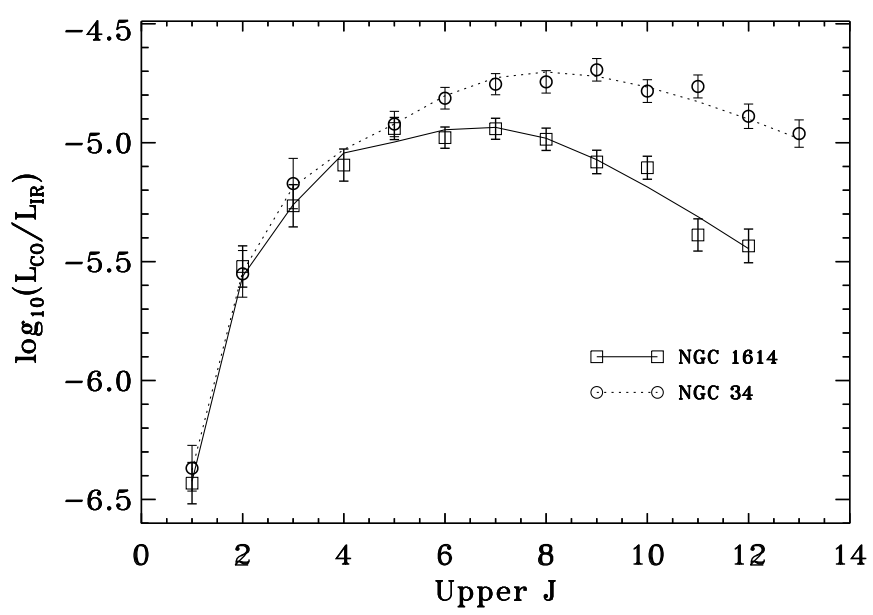

Figure 9. Plot of the $L_{\mathrm{IR}}$ normalized spectral line energy distributions (SLEDs) of NGC 1614 and NGC 34. The data points (all obtained by single-dish observations) are taken from the literature with the following references: for NGC 1614: Sanders et al. (1991) for CO (1-0); Albrecht et al. (2007) for CO (2-1); Wilson et al. (2008) for CO (3-2); N. Lu et al. (2014, in preparation) for CO (4-3) and other higher-J lines; for NGC 34: (Albrecht et al. 2007) and Maiolino et al. (1997) for CO (1-0); Papadopoulos \& Seaquist (1998) for CO (2-1); Q. Zhang et al. (2014, in preparation) for CO (3-2); N. Lu et al. (2014, in preparation) for $\mathrm{CO}(4-3)$ and other higher- $J$ lines. The solid (dotted) line is model fitting of the CO SLED of NGC 1614 (NGC 34).

these conversion factors are very uncertain and are the major error sources for the molecular gas mass estimates.

In Figure 9, we compare the spectral line energy distributions (SLEDs) of the total CO emission (measured by single-dish observations) of these two galaxies, taken from observations of Herschel-SPIRE FTS observations (N. Lu et al. 2014, in preparation). The CO SLED of NGC 1614 peaks around upper $J=5-7$, while that of NGC 34 reaches a plateau after a rapid increase, and the peak is around upper $J=9$. In order to further investigate the physical conditions of these two galaxies, we modeled the observed CO SLEDs using simple two-component RADEX large velocity gradient (LVG) radiative transfer models (van der Tak et al. 2007), and adopting a similar procedure to that in Kamenetzky et al. (2012). Admittedly, results from such model fittings suffer significant degeneracy between parameters (Rosenberg et al. 2014a). Nevertheless, they are useful for translating the information in the CO SLED into quantitative estimates of physical parameters of the gas, albeit with large uncertainties. We find that both SLEDs can be well fit by the combination of a cool and a warm component. Both galaxies have similar gas densities of $\left(10^{2.5}, 10^{2.6}\right) \mathrm{cm}^{-3}$ and $\left(10^{4}, 10^{4}\right) \mathrm{cm}^{-3}$, for the cool and warm components in (NGC 34, NGC 1614), respectively. However, the kinetic temperature of the warm component in NGC $34(890 \mathrm{~K})$ is two times higher than that of NGC 1614 (445 K), consistent with the fact that the nuclear starburst in NGC 34 is five times more compact than the circumnuclear starburst ring in NGC 1614. It is worthwhile noting that the AGN contribution to the warm gas in NGC 34 is insignificant ( $\mathrm{Xu}$ et al. 2014). Given the overall similarities between the two host galaxies (Table 3), it is likely that the staunch difference between the two central starbursts is caused by the difference in the merging processes that the two LIRGs have experienced.

The morphology of NGC 1614-one prominent tail and one relatively small secondary tail-suggests an unequal mass encounter (mass ratio $\gtrsim 4: 1$ ) and/or a scenario in which one of the galaxies experienced a retrograde passage. Several 
authors have argued for a high mass ratio encounter; Rothberg \& Joseph (2006) note the isophotal shape of NGC 1614 and its correspondence with simulations of high mass ratio mergers, while Väisänen et al. (2012) identify a possible remnant body of the lower-mass companion. Both Rothberg \& Joseph (2006) and Väisänen et al. (2012) come to the same conclusion-that NGC 1614 is a 4:1 mass ratio merger-but the former assumes the nuclei have already merged and the latter relies on the identification of an interacting galaxy.

NGC 34 has no clear evidence for dual nuclei, suggesting the two galaxies have already coalesced. Owing to the asymmetry of integrated brightness of the two tidal tails Schweizer \& Seitzer (2007) argue that this system is the result of a merger of two disk galaxies with a mass ratio between $3: 2$ and $3: 1$. The disky isophotal shape of the remnant (which shows no evidence for a disk in the $K$-band morphology) is consistent with a formation scenario of a major but unequal mass merger (Rothberg \& Joseph 2006; Naab et al. 2006). Preliminary dynamical modeling of this system is consistent with the aforementioned mass ratio and both disks experiencing prograde interactions (G. C. Privon et al., in preparation). This dynamical model is consistent with the system being observed $\sim 250-300$ Myr since the first passage of the two galaxies, somewhat lower than the suggested $400 \mathrm{Myr}$ age of the stellar disk (Schweizer \& Seitzer 2007).

Hence, NGC 34 has experienced a major merger of two galaxies of similar mass, which was catastrophic and destroyed both progenitor disks (Schweizer \& Seitzer 2007). Simulations by Cox et al. (2008) exploring the effect of mass ratio on merger-induced starbursts found a decreasing burst strength with increasing primary/secondary mass ratio; given the previously mentioned estimates of the mass ratios for NGC 34 and NGC 1614, the star formation surface densities are consistent with this interpretation. It might be that the higher mass ratio merger experienced by NGC 1614 caused less efficient torquing of the gas, leading to much of the central gas settling into the nuclear ring (with the help of either the inner Lindblad resonance associated with a bar (Olsson et al. 2010) or the non-axisymmetric potential caused by a minor merger (Combes 1988; Knapen et al. 2004; Mazzuca et al. 2006)) rather than collecting in the center, as in NGC 34. This may also hint at the answer to the question why NGC 1614 has not yet developed an AGN (Väisänen et al. 2012) while NGC 34 has. According to Hopkins (2012), the built-up of a centrally peaked dense gas disk is a necessary condition for triggering of the AGN activity in late stage mergers.

An alternate explanation for NGC 1614's comparatively lower $\Sigma_{\mathrm{SFR}}$, if the scenario proposed by Väisänen et al. (2012) is accurate, is that the merger has simply not yet run to completion and so has not yet caused the final funneling of gas toward the nucleus at the time of the merger (e.g., Mihos \& Hernquist 1994; Hopkins 2012). Olsson et al. (2010) and König et al. (2013) both show that indeed most of the molecular gas in NGC 1614 sits in the dust lane (outside the ring) and even further out. It could be that the relatively minor perturbation of the first pass (which led to the northeast tail) created the outward propagating starburst (i.e., the "wild fire"), as revealed by the nuclear ring and the weak and old nuclear starburst, while a future merger will trigger a much stronger nuclear starburst as seen in NGC 34.

With current knowledge of the encounters in NGC 34 and NGC 1614, we cannot firmly assign the cause for the different starburst characteristics in the two systems. It is likely to be due to the effect of different mass ratios, but we cannot rule out other possible causes such as different current phases of the encounters and different encounter geometries. While NGC 34 represents a large population of LIRGs with starburst nuclei (e.g., Arp 220), NGC 1614 represents those with circumnuclear starburst rings, which are also common in LIRGs. Among the GOALS sample, at least five other LIRGs (NGC 1068, NGC 5135, NGC 7469, NGC 7552, and NGC 7771) have such rings. Future dynamical models (G. C. Privon et al., in preparation) matched to the kinematics and morphology of NGC 34 and NGC 1614 may provide a more concrete answer to the question of how the two galaxies, and the two LIRG populations they represent, developed such different central starbursts over the merging process.

\section{SUMMARY}

We carried out ALMA observations of the CO (6-5) line emission and of the $435 \mu \mathrm{m}$ dust continuum emission in the central kiloparsec of NGC 1614, a local LIRG at distance of $67.8 \mathrm{Mpc}\left(1^{\prime \prime}=329 \mathrm{pc}\right)$. The $\mathrm{CO}$ emission and the continuum are both well resolved by the ALMA beam $(0.26 \times 0.20)$ into a circumnuclear ring. The integrated flux of $\mathrm{CO}(6-5)$ is $f_{\mathrm{CO}(6-5)}=898( \pm 153) \mathrm{Jy} \mathrm{km} \mathrm{s}{ }^{-1}$, and the flux of the continuum is $f_{\mathrm{CO}(6-5)}=269( \pm 46) \mathrm{mJy}$. These are $63( \pm 12) \%$ and $32( \pm 6) \%$ of the total $\mathrm{CO}(6-5)$ flux and $435 \mu \mathrm{m}$ continuum flux of NGC 1614 measured by Herschel, respectively. The molecular ring, located between $100 \mathrm{pc}<\mathrm{r}<350 \mathrm{pc}$, looks clumpy and includes several unresolved (or marginally resolved) knots with median velocity dispersion of $\delta \mathrm{v} \sim 40 \mathrm{~km} \mathrm{~s}^{-1}$. These knots are associated with star formation regions with $\Sigma_{\mathrm{SFR}} \sim 100 M_{\odot} \mathrm{yr}^{-1} \mathrm{kpc}^{-2}$ and $\Sigma_{\mathrm{Gas}} \sim 10^{4} M_{\odot} \mathrm{pc}^{-2}$. The nondetections of the nucleus in both the $\mathrm{CO}(6-5)$ and the $435 \mu \mathrm{m}$ continuum rule out, with relatively high confidence, a Comptonthick AGN in NGC 1614. Comparisons with the radio continuum show that the local correlation, on the linear scale of $\sim 100 \mathrm{pc}$, between $\Sigma_{\mathrm{Gas}}$ and $\Sigma_{\mathrm{SFR}}$ (i.e., the Kennicutt-Schmidt law) is severely disturbed. In particular, the nucleus has a lower limit of the $\Sigma_{\mathrm{SFR}}$-to- $\Sigma_{\mathrm{Gas}}$ ratio about an order of magnitude above the nominal value in the standard Kennicutt-Schmidt law. This breakdown of the star formation law could be caused by an outward propagation of the central starburst (i.e., the "wild fire" scenario proposed by Alonso-Herrero et al. 2001). Our results also show that the $\mathrm{CO}(6-5)$ correlates stronger with the nonthermal radio component than both the total radio emission and the thermal radio component, possibly due to an in situ correlation between the magnetic field strength and the gas density.

We thank Adam Leroy and Tony Remijan from NAASC for their help with data reduction. We thank an anonymous referee for constructive comments. Y.G. is partially supported by NSFC-11173059, NSFC-11390373, and CASXDB09000000. Y.Z. thanks the NSF of Jiangsu Province for partial support under grant BK2011888. V.C. acknowledges partial support from the EU FP7 grant PIRSES-GA-2012316788. This paper makes use of the following ALMA data: ADS/JAO.ALMA\#2011.0.00182.S. ALMA is a partnership of ESO (representing its member states), NSF (USA), and NINS (Japan), together with NRC (Canada) and NSC and ASIAA (Taiwan), in cooperation with the Republic of Chile. The Joint ALMA Observatory is operated by ESO, AUI/NRAO, and NAOJ. This research has made extensive use of the NASA/ IPAC Extragalactic Database (NED) which is operated by the Jet Propulsion Laboratory, California Institute of Technology, 


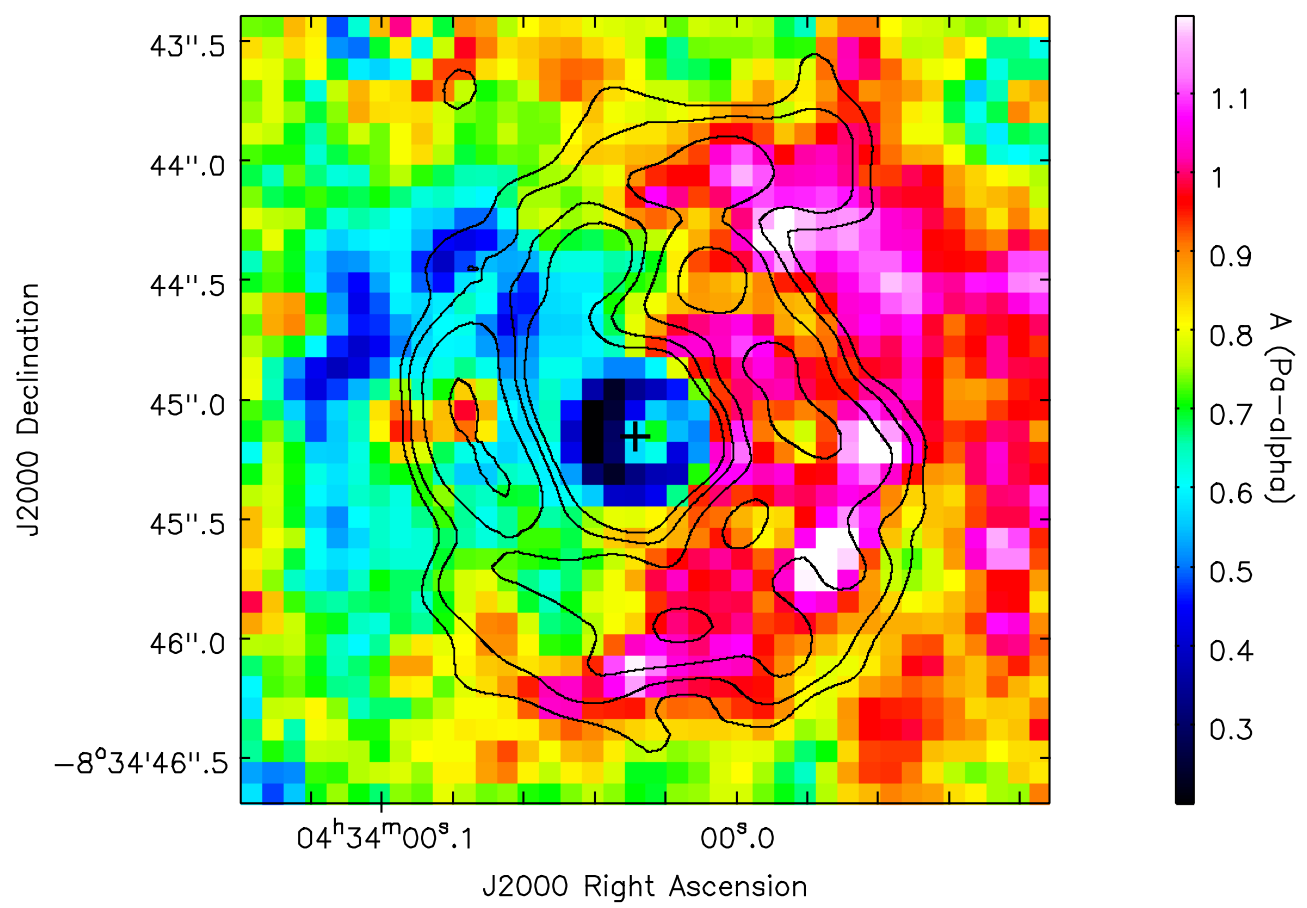

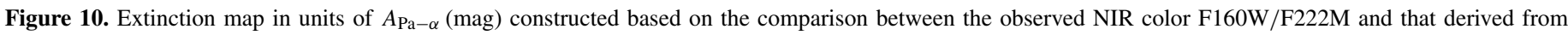
Starburst99 models for the stellar population ages estimated from the Pa- $\alpha$ EW map. Contours of the CO (6-5) line emission are overlaid on the map.

under contract with the National Aeronautics and Space Administration.

\section{APPENDIX}

\section{THERMAL/NONTHERMAL DECOMPOSITION OF THE RADIO CONTINUUM}

The thermal radio emission is estimated from an extinctioncorrected Pa- $\alpha$ map of the central region of NGC 1614 derived from HST NICMOS imaging data (Alonso-Herrero et al. 2001) taken in four NIR continuum and emission line bands (F160W and F222M, and F187N and F190N, respectively). The maps are background-subtracted (Díaz-Santos et al. 2008). The dustattenuated $\mathrm{Pa}-\alpha$ emission map was obtained from the F190N narrow-band line+continuum image after the subtraction of the adjacent continuum emission obtained with the F187N filter. In order to correct the $\mathrm{Pa}-\alpha$ map for the significant extinction (e.g., Neff et al. 1990; Alonso-Herrero et al. 2001; Kotilainen et al. 2001; Rosenberg et al. 2012), we used the EW map of the emission line plus two broadband NIR continuum images obtained at 1.6 and 2.2 um.

First, we used Starburst99 (v7.0.0; Leitherer et al. 1999) to generate a stellar population synthesis model for an instantaneous burst of star formation with Geneva evolutionary stellar tracks, Kroupa initial mass function, and solar metallicity. The model outputs were obtained with a $0.1 \mathrm{Myr}$ step for starburst ages ranging from 0.01 to $50 \mathrm{Myr}$. In addition to the spectral energy distribution (SED) of the continuum, the model also provides the nebular emission from hydrogen recombination lines. Assuming that the extinction to the gas is similar to that toward the dust, the observed $\mathrm{Pa}-\alpha \mathrm{EW}$ map was compared to the model predictions to estimate the age of the young stellar population. We note that the ages are upper limits to the real ages since the presence of an older, underlying stellar population would increase the NIR continuum, thus aging the regions.
Once the ages of the stellar populations are estimated, we can compare the NIR continuum slope derived from the two continuum broadband filter images with the model SEDs to derive the obscuration. To this end, the Starburst99 spectra were convolved for each age step with the corresponding continuum filters. The synthetic NIR colors (F160W/F222M) were interpolated to the ages derived from the $\mathrm{Pa}-\alpha \mathrm{EW}$ map and compared with the observed values, and the extinction was estimated from this comparison. The dust attenuation law used to derive the extinction was that of Calzetti et al. (2000) for a foreground dust screen configuration. The resulting extinction map in units of $A_{\mathrm{Pa}-\alpha}$ (mag) is shown Figure 10. The typical error of $A_{\mathrm{Pa}-\alpha}$ (mag) is $0.3 \mathrm{mag}$, estimated from the uncertainties of the age and the color map. The strong east-west asymmetry in the $A_{\mathrm{Pa}-\alpha}$ map is in agreement with that seen in the low-J CO maps (Olsson et al. 2010; König et al. 2013), and is likely associated with a broad dust-lane extended from the north to the west of the ring (König et al. 2013). Finally, the observed Pa- $\alpha$ image was corrected using the obscuration map obtained from this method. The extinction-corrected $\mathrm{Pa}-\alpha$ emission was then scaled to obtain the thermal component of the radio emission at 8.4 GHz (see Equation (4) in Herrero-Illana et al. 2014). The nonthermal component was then obtained by subtracting the thermal emission from the original radio map.

\section{REFERENCES}

Aalto, S., Garcia-Burillo, S., Muller, S., et al. 2012, A\&A, 537, A44 Albrecht, M., Krügel, E., \& Chini, R. 2007, A\&A, 462, 575

Alonso-Herrero, A., Engelbracht, C. W., Rieke, M. J., et al. 2001, ApJ, 546, 952

Armus, L., Heckman, T. M., \& Miley, G. K. 1989, ApJ, 347, 727

Armus, L., Heckman, T. M., \& Miley, G. K. 1990, ApJ, 364, 471

Armus, L., Mazzarella, J. M., Evans, A. S., et al. 2009, PASP, 121, 599

Barnes, J., \& Hernquist, L. 1996, ApJ, 471, 115

Barnes, J. E. 1990, Natur, 344, 379

Bell, E. F., Zheng, X. Z., Papovich, C., et al. 2007, ApJ, 663, 834

Brightman, M., \& Nandra, K. 2011, MNRAS, 413, 1206

Bryant, P. M., \& Scoville, N. Z. 1999, AJ, 117, 2632 
Calzetti, D., Armus, L., Bohlin, R. C., et al. 2000, ApJ, 533, 682 Casoli, F., Dupraz, C., Combes, F., \& Kazes, I. 1991, A\&A, 251, 1 Cicone, C., Maiolino, R., Sturm, E., et al. 2014, A\&A, 562, A21

Comastri, A. 2004, in Supermassive Black Holes in the Distant Universe, ed A. J. Barger (Astrophysics and Space Science Library, Vol. 308; Dordrecht: Kluwer), 245

Combes, F. 1988, in NATO ASIC Proc. 232: Galactic and Extragalactic Star Formation, ed. R. E. Pudritz \& M. Fich (Dordrecht: Kluwer), 475

Condon, J. J., Huang, Z.-P., Yin, Q.-F., \& Thuan, T. 1991, ApJ, 378, 65

Costagliola, F., Aalto, S., Rodriguez, M. I., et al. 2011, A\&A, 528, A30

Cox, T. J., Jonsson, P., Somerville, R. S., Primack, J. R., \& Dekel, A. 2008, MNRAS, 384, 386

Daddi, E., Elbaz, D., Walter, F., et al. 2010, ApJL, 714, 118

Dasyra, K. M., Tacconi, L. J., Davies, R. I., et al. 2006, ApJ, 638, 745

Díaz-Santos, T., Alonso-Herrero, A., Colina, L., et al. 2008, ApJ, 685, 211

Downes, D., \& Solomon, P. M. 1998, ApJ, 507, 615

Draine, B. T. 1980, ApJ, 241, 1021

Draine, B. T., \& Li, A. 2007, ApJ, 657, 810

Drury, L. O., Aharonian, F. A., \& Voelk, H. J. 1994, A\&A, 287, 959

Engelbracht, C. W., Rieke, G. H., Gordon, K. D., et al. 2008, ApJ, 678, 804

Esquej, P., Alonso-Herrero, A., Perez-Garcia, A. M., et al. 2012, MNRAS, 423,185

Evans, A. S., Mazzarella, J. M., Surace, J. A., \& Sanders, D. B. 2002, ApJ, 580,749

Faber, S. M., Willmer, C. N. A., Wolf, C., et al. 2007, ApJ, 665, 265

Fernandez, X., Petric, A. O., Schweizer, F., \& van Gorkom, J. H. 2014, AJ, 147, 74

Feruglio, C., Maiolino, R., Piconcelli, E., et al. 2010, A\&A, 518, L155

Fiebig, D., \& Guesten, R. 1989, A\&A, 214, 333

Fischer, J., Sturm, E., González-Alfonso, E., et al. 2010, A\&A, 518, L41

Gao, Y., Lo, K. Y., Lee, S.-W., \& Lee, T.-H. 2001, ApJ, 548, 172

Gao, Y., \& Solomon, P. M. 1999, ApJL, 512, L99

Gao, Y., \& Solomon, P. M. 2004a, ApJS, 152, 63

Gao, Y., \& Solomon, P. M. 2004b, ApJ, 606, 271

García-Burillo, S., Combes, F., Usero, A., et al. 2014, A\&A, 567, A125

García-Burillo, S., Usero, A., Alonso-Herrero, A., et al. 2012, A\&A, 539, A8

Genzel, R., Lutz, D., Sturm, E., et al. 1998, ApJ, 498, 579

Genzel, R., Tacconi, L. J., Gracia-Carpio, J., et al. 2010, MNRAS, 407, 2091

Genzel, R., Tacconi, L. J., Rigopoulou, D., Lutz, D., \& Tecza, M. 2001, ApJ, 563,527

Graciá-Carpio, J., García-Burillo, S., Planesas, P., et al. 2008, A\&A, 479, 703

Griffin, M. J., Abergel, A., Abreu, A., et al. 2010, A\&A, 518, L3

Hailey-Dunsheath, S., Nikola, T., Stacey, G. J., et al. 2008, ApJL, 689, L109

Heckman, T. M., Lehnert, M. D., Strickland, D. K., \& Armus, L. 2000, ApJS, 129,493

Helou, G., \& Bicay, M. D. 1993, ApJ, 415, 93

Herrero-Illana, R., Pérez-Torres, M. Á., Alonso-Herrero, A., et al. 2014, ApJ, 786, 156

Hopkins, P. F. 2012, MNRAS, 420, L8

Hopkins, P. F., Cox, T. J., Kere, D., \& Hernquist, L. 2008, ApJS, 75, 390

Hopkins, P. F., Cox, T. J., Younger, J. D., \& Hernquist, L. 2009, ApJ, 691, 1186

Hopkins, P. F., Kereš, D., Murray, N., et al. 2013, MNRAS, 433, 78

Imanishi, M., \& Nakanishi, K. 2013, AJ, 146, 47

Iono, D., Ho, P. T. P., Yun, M. S., et al. 2004, ApJL, 616, L63

Iono, D., Wilson, C. D., Yun, M. S., et al. 2009, ApJ, 695, 1537

Kamenetzky, J., Glenn, J., Rangwala, N., et al. 2012, ApJ, 753, 70

Kandalyan, R. A. 2003, A\&A, 398, 493

Kazandjian, M. V., Meijerink, R., Pelupessy, I., et al. 2012, A\&A, 542, A65

Kazandjian, M. V., Meijerink, R., Pelupessy, I., et al. 2014, arXiv:1403.7000

Kennicutt, R. C. 1998, ApJ, 498, 541

Knapen, J. H., Whyte, L. F., de Blok, W. J. G., et al. 2004, A\&A, 423, 481

König, S., Aalto, S., Muller, S., Beswick, R. J., \& Gallagher, J. S. 2013, A\&A, 553, A72

Kotilainen, J. K., Reunanen, J., Laine, S., \& Ryder, S. D. 2001, A\&A, 366, 439

Kruegel, E., Chini, R., \& Steppe, H. 1990, A\&A, 229, 17

Leitherer, C., Schaerer, D., Goldader, J. D., et al. 1999, ApJS, 123, 3

Leroy, A. K., Walter, F., Sandstrom, K., et al. 2013, AJ, 146, 19

Lu, N., Zhao, Y., Xu, C. K., et al. 2014, ApJL, 787, L23
Maiolino, R., Ruiz, M., Rieke, G. H., \& Papadopoulos, P. 1997, ApJ, 485, 552

Mazzuca, L. M., Sarzi, M., Knapen, J. H., et al. 2006, ApJL, 649, L79

Meijerink, R., \& Spaans, M. 2005, A\&A, 436, 397

Mihos, J. C., \& Hernquist, L. 1994, ApJL, 431, L9

Modjaz, M., Kewley, L., Bloom, J. S., et al. 2011, ApJL, 731, L4

Murphy, E. J., Bremseth, J., Mason, B. S., et al. 2012, ApJ, 761, 97

Naab, T., Jesseit, R., \& Burkert, A. 2006, MNRAS, 372, 839

Narayanan, D., Cox, T. J., Shirley, Y., et al. 2008, ApJ, 684, 996

Neff, S. G., Hutchings, J. B., Standord, S. A., \& Unger, S. W. 1990, AJ, 99, 1088

Niklas, S., \& Beck, R. 1997, A\&A, 320, 54

Olsson, E., Aalto, S., Thomasson, M., \& Beswick, R. 2010, A\&A, 513, A11

Onodera, S., Kuno, N., Tosaki, T., et al. 2010, ApJL, 722, L127

Papadopoulos, P. P., \& Seaquist, E. R. 1998, ApJ, 492, 521

Papadopoulos, P. P., van der Werf, P. P., Xilouris, E. M., et al. 2012, MNRAS, 426, 2601

Rémy-Ruyer, A., Madden, S. C., Galliano, F., et al. 2014, A\&A, 563, A31

Risaliti, G., Gilli, R., Maiolino, R., \& Salvati, M. 2000, A\&A, 357, 13

Rosenberg, M. J. F., Kazandjian, M. V., van der Werf, P. P., et al. 2014a, A\&A, 564, A126

Rosenberg, M. J. F., Meijerink, R., Israel, F. P., et al. 2014b, A\&A, 568, 90

Rosenberg, M. J. F., van der Werf, P. P., \& Israel, F. P. 2012, A\&A, 540, A116

Rothberg, B., \& Joseph, R. D. 2004, AJ, 128, 2098

Rothberg, B., \& Joseph, R. D. 2006, AJ, 132, 976

Rupke, D. S., Veilleux, S., \& Sanders, D. B. 2005, ApJS, 160, 115

Sakamoto, K., Aalto, S., Costagliola, F., et al. 2013, ApJ, 764, 42

Sakamoto, K., Aalto, S., Wilner, D. J., et al. 2009, ApJL, 700, L104

Sakamoto, K., Wang, J., Wiedner, M. C., et al. 2008, ApJ, 684, 957

Sanders, D. B., \& Mirabel, I. F. 1996, ARA\&A, 34, 749

Sanders, D. B., Scoville, N. Z., \& Soifer, B. T. 1991, ApJ, 370, 158

Sanders, D. B., Soifer, B. T., Elias, J. H., et al. 1988, ApJ, 325, 74

Schmitt, H. R., Calzetti, D., Armus, L., et al. 2006, ApJS, 164, 52

Schweizer, F. 1982, ApJ, 252, 455

Schweizer, F., \& Seitzer, P. 2007, AJ, 133, 2132

Scoville, N. Z., Evans, A. S., Thompson, R., et al. 2000, AJ, 119, 991

Scoville, N. Z., Sanders, D. B., Sargent, A. I., et al. 1989, ApJL, 345, L25

Scoville, N. Z., Yun, M. S., \& Bryant, P. M. 1997, ApJ, 484, 702

Soifer, B. T., Neugebauer, G., Matthews, K., et al. 2001, AJ, 122, 1213

Solomon, P. M., Downes, D., \& Radford, S. J. E. 1992, ApJL, 387, L55

Solomon, P. M., Downes, D., Radford, S. J. E., \& Barrett, J. W. 1997, ApJ, 478,144

Solomon, P. M., \& Sage, L. J. 1988, ApJ, 334, 613

Stierwalt, S., Armus, L., Surace, J. A., et al. 2013, ApJS, 206, 1

Sturm, E., González-Alfonso, E., Veilleux, S., et al. 2011, ApJL, 733, 16

Surace, J. A., Sanders, D. B., Vacca, W. D., et al. 1998, ApJ, 492, 116

Toomre, A. 1977, in Evolution of Galaxies and Stellar Populations, ed. B. M

Tinsley, R. B. G. Larson, \& D. Campbell (New Haven, CT: Yale University Observatory), 401

Tsai, M., Hwang, C.-Y., Matsushita, S., et al. 2012, ApJ, 746, 129

U, V., Sanders, D. B., Mazzarella, J. M., et al. 2012, ApJS, 203, 9

Vacca, W. D., \& Conti, P. S. 1992, ApJ, 401, 543

Väisänen, P., Rajpaul, V., Zijlstra, A. A., et al. 2012, MNRAS, 420, 2209

Vallée, J. P. 1990, A\&A, 239, 57

Vallée, J. P. 1995, Ap\&SS, 234, 1

van der Tak, F. F. S., Black, J. H., Schöier, F. L., et al. 2007, A\&A, 468, 627

van der Werf, P. P., Isaak, K. G., Meijerink, R., et al. 2010, A\&A, 518, L42

Veilleux, S., Kim, D.-C., \& Sanders, D. B. 1999, ApJ, 522, 113

Veilleux, S., Kim, D.-C., \& Sanders, D. B. 2002, ApJS, 143, 315

Veilleux, S., Melendez, M., Sturm, E., et al. 2013, ApJ, 776, 27

Veilleux, S., Rupke, D. S. N., Kim, D.-C., et al. 2009, ApJS, 182, 628

Walter, F., Weiss, A., \& Scoville, N. 2002, ApJL, 580, L21

Wang, J., Zhang, Q., Wang, Z., et al. 2004, ApJL, 616, L67

Wilson, C. D., Petitpas, G. R., Iono, D., et al. 2008, ApJS, 178, 189

Wootten, A., \& Thompson, A. R. 2009, IEEEP, 97, 1463

Xu, C. K., Cao, C., Lu, N., et al. 2014, ApJ, 787, 48

Yao, L., Seaquist, E. R., Kuno, N., \& Dunne, L. 2003, ApJ, 588, 771

Young, J. S., Kenney, J. D., Tacconi, L., et al. 1986, ApJL, 311, L17 